\title{
Integral Decomposition of Partial *-Algebras of Closed Operators
}

\author{
By
}

\author{
Françoise D. MATHOT*
}

\begin{abstract}
A method of direct integral decomposition for very general classes of unbounded closed operators is developed. This method is applied to the reduction theory of partial *-algebras and $\mathrm{Op}^{*}$-algebras.

As a consequence, representations of partial $*_{\text {-algebras are decomposed into irreducible }}$ ones and some states are decomposed into extremal states.
\end{abstract}

\section{Introduction}

In this paper, we present a method of integral decomposition for some families of unbounded operators in a Hilbert space. The sets of operators we consider are fairly general. They consists of closed operators with a common core $\mathscr{D}$ (dense in $\mathscr{H}$ ) and we only ask our sets to be invariant under some involution. In particular, the operators need not leave the domain invariant.

Important examples of such sets are given by partial *-algebras (first introduced by Borchers) [1] and developed systematically by Antoine-Karwowski [2] [3] and Op*-algebras which have been extensively studied by Lassner and his group [4], Vasil'ev [5], Powers [6], Epifanio [7]. In the last category of examples, the domain $\mathscr{D}$ is left invariant and products between operators are always defined.

However, in some situations, the use of Op*-algebras is not sufficient. For instance, there exist some quantum statistical systems for which the thermodynamical limit does not exist in a $C^{*}$-topology

Communicated by H. Araki, November 30, 1985.

* Institut de Physique Théorique, Université Catholique de Louvain, B-1348 Louvain-laNeuve, Belgique. 
[8] but only in the completion of some Op*-algebra. This completion, called a quasi-algebra [9], is no longer an algebra itself, but a more general structure where the product between two elements need not be defined.

This fact and some other examples (generalized creation and annihilation operators [10], use of potentials which do not preserve the invariant domain for the observables in Quantum Mechanics [11]) have motivated the systematic study of the partial *-algebras [2], of their states and representations [3], [12]. This motivates also the present work.

As long as bounded operators are concerned, the problem of integral decomposition is well known and it gave rise to the reduction theory for von Neumann algebras [13]. For unbounded operators, several authors developed different methods of decomposition.

Nussbaum [14] has considered closed operators and has related their decomposability in a direct integral to the decomposability of their characteristic matrix (essentially a $2 \times 2$ matrix obtained from the projection in $\mathscr{H} \times \mathscr{H}$ onto the graph of the closed operator). He also characterized the operators which may be decomposed in a direct integral of bounded operators.

Borchers and Yngvason [15] have considered families of unbounded operators defined on a common nuclear domain. By the nuclear spectral theorem they could define in each $\mathscr{H}(\lambda)$, occurring in the decomposition of the Hilbert space, a dense domain $\mathscr{D}(\lambda)$, also nuclear, on which all the $A(\lambda)$, coming from the decomposition of the initial operators $A$, are defined.

They applied their method to the decomposition of representations

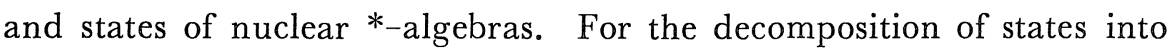
extremal states, the Choquet decomposition theory has been used by Hegerfeld [16], also for nuclear $*_{\text {-algebras. }}$

In a previous work [17] [18], we have studied the decomposition of Op*-algebras defined on a metrizable domain. Our method consisted in the identification of an unbounded operator with a family of bounded operators between different Hilbert spaces. We then decomposed simultaneously all the present Hilbert spaces and the bounded operators between them. Conversely, the family of bounded operators we obtain in each $\lambda$-component of the direct integral, allowed us to 
reconstruct an unbounded operator $A(\lambda)$ in $\mathscr{H}(\lambda)$. The same kind of method will be applied here for partial $*_{-}$algebras and more general families.

The assumption of metrizability of $\mathscr{D}$ occurs often in the literature. Interesting properties about topologies [19], states [20], commutants [21] have been established in that situation.

In our problem of integral decomposition, this hypothesis allowed us, for Op*-algebras, to define a common invariant dense domain $\mathscr{D}(\lambda)$ in $\mathscr{H}(\lambda)$ on which $\{A(\lambda)\}$ coming from the decomposition of the Op*-algebra, forms itself an Op*-algebra. (Actually, the metrizability of $\mathscr{D}$ prevents us from having to deal with an uncountable union of null-sets.) For the same reason, we assume all along this paper that $\mathscr{D}$ is metrizable. Although it will be more difficult in our case to define the domain $\mathscr{D}(\lambda)$ (see Section 3 ) than for Op*-algebras, the metrizability of $\mathscr{D}$ will be essential to build it and to prove that it is dense in $\mathscr{H}(\lambda)$ and a core for each $A(\lambda)$. It will also be important in the verification that the algebraic operations (which are very specific for partial *-algebras), go through the integral decomposition.

The first part of the paper is devoted to the decomposition of a countable set of operators (this automatically gives us a metrizable domain) and is organized as follows:

Section $\mathbb{I}$ : Definitions and preliminaries: $\neq$-invariant sets, partial *-algebras, Op*-algebras, graph-topology, bounded and unbounded commutants.

Section II: Integral decomposition of a single operator: Decomposition of $\mathscr{H}$ and of the domain of the operator, characterization of the decomposable operators.

Section $\mathbb{I l}$ : Construction of a common domain $\mathscr{D}(\lambda)$ for a countable set of operators: metrizability condition on $\mathscr{D}$, existence of $\mathscr{D}(\lambda)$.

Section IV: Verification of the algebraic properties: Adjoint, sum, products (as defined in partial *-algebras)

The conclusion of this first part is that any countable $\neq-$ invariant set (resp. partial *-algebra or Op*-algebra) may be decomposed in a direct integral of countable irreducible $\neq-$ invariant sets (resp. in 
partial $*_{\text {-algebras }}$ or in $\mathrm{Op}^{*}$-algebras $)$ on a domain $\mathscr{D}(\lambda)$ of $\mathscr{H}(\lambda)$.

The second part of the paper is devoted to the extension of this result to non-countable but separable $\neq$-invariant sets.

In the case of bounded operators [13], this extension is possible because a von Neumann algebra is separable in the strong operator topology and because if $A^{i} \rightarrow A$ and $\left\{A^{i}\right\}$ and $A$ are all decomposable, then $A^{i}(\lambda) \rightarrow A(\lambda)$ for almost every $\lambda$ (i.e. $A(\lambda)$ belongs to the von Neumann algebra generated by $\left.\left\{A^{i}(\lambda)\right\}\right)$.

The main point here is to find a suitable topology (involving the strong one in some sense) and assume the family of operators is separable in this topology. For Op*-algebras we considered in [18] a quasi-uniform topology [4], [22] and then proved the result that separable Op*-algebras could be decomposed.

Here, we can actually consider a weaker topology, the strong *-topology ([23] for bounded operators, [24] for unbounded) which is a particular case of a quasi-uniform topology. In practice, when we shall consider representations of abstract partial *-algebras by closed operators, the assumption of separability in the strong *-topology will come from the fact that we shall consider strongly continuous representations.

In the second part of this paper, we decompose thus $\neq-$ invariant

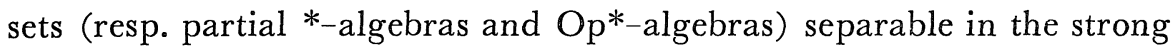

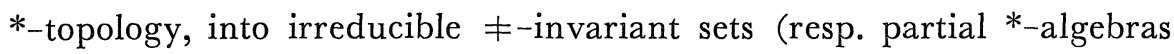
and $\mathrm{Op}^{*-a l g e b r a s) . ~ F o r ~} \mathrm{Op}^{*}$-algebras this generalizes the result we got in [18].

We also apply this decomposition method to some particular classes: the $V^{*}$-sets which are sets of operators equal to their unbounded bicommutant (this is a generalization of the $V^{*}$-algebras considered by [25] which are themselves a generalization of von Neumann algebras).

Finally, we decompose representations of abstract partial *-algebras into irreducible representations and states into extremal states. Concerning the last point, the usual notion of state on a *-algebra has to be replaced by sesquilinear forms, the so-called $h$-states [3]. Some of them, called weakly GNS $h$-states give rise to a generalization of the GNS representation and these are the ones we decompose in this 
paper.

The second part is organized as follows:

Section $\mathbb{V}$ : Decomposition of separable $\neq-$-invariant sets: Strong *-topology, separability, decomposition of the dense subset $\mathfrak{X}_{0}$, topology on $\mathfrak{Y}_{0}(\lambda)$, completion of $\mathfrak{U}_{0}(\lambda)$, decomposition of the whole set. Particular cases: $V^{*}$-sets and $V^{*}$-algebras.

Section $\mathbf{V}$ : Decomposition of representations of partial *-algebras: Abstract partial *-algebras, representations, closed and fully closed representations, cyclic and strongly cyclic vectors. Theorem of decomposition into irreducible representations. Continuity property of the irreducible representations.

Section $\mathrm{VIII}$ : Decomposition of states of partial *-algebras: Definitions of $h$-forms, $h$-states, weakly GNS $h$-states. Theorem of decomposition of weakly GNS $h$-states into extremal ones. Continuity property of extremal h-states.

\section{Acknowledgements}

It is a pleasure to express here my gratitude to Professor J. $\mathbb{P}$. Antoine for mathematical discussions about this work and for his appreciated linguistic help.

\section{Part one: Decomposition of a Countable Set of Operators}

\section{$\S \mathbb{I}$. Definitions and Preliminaries}

\section{1. $\neq$-invariant sets, partial *-algebras, Op*-algebras}

Throughout this paper $\mathscr{H}$ will denote a separable Hilbert space and $\mathscr{D}$ a dense domain in it.

We shall deal with a family $\mathfrak{A}$ of closed linear operators having $\mathscr{D}$ as a common core (but not necessarily leaving it invariant).

According to the definitions of [2], $\mathfrak{A}$ is thus a subset of the set $\sqrt{5}$ or $\mathfrak{C}(\mathscr{D})$ of $\mathscr{D}$-minimal operators i.e. closed operators $A$ in $\mathscr{H}$ such that $\mathscr{D} \subseteq D(A) \cap D\left(A^{*}\right)$ and satisfying $A=\overline{A \uparrow \mathscr{D}}$ (where * denotes the usual Hilbertian adjoint and the bar, the usual closure of an operator). 
The set $\mathfrak{E}$ carries a natural involution $\neq$ defined by: $A^{+} \equiv \overline{A^{*}\lceil\mathscr{D}}$. Moreover, every element $A$ of $\sqrt{5}$ satisfies $A=A^{\neq \neq}$.

The kind of families we are going to consider in the sequel of this paper will be $\neq$-invariant subsets of $\mathfrak{c}$. We shall refer to those subsets as " $\neq$-invariant sets" (of $\mathscr{D}$-minimal closed operators).

Important particular cases are partial *-algebras [2] and Op*algebras [4]. For convenience of the reader we recall the basic algebraic notions involved in those structures.

a) If $A, B \in \mathfrak{C}$ the sum $A \hat{+} B \equiv \overline{(A+B)\lceil\mathscr{D}}$ belongs to $\mathbb{E}$ again.

b) Two kinds of partial multiplication may be introduced:

Let $A$ and $B$ be such that $B \mathscr{D} \subseteq D(A)$ (domain of $A$ ) and $A^{*} \mathscr{D} \subseteq D\left(B^{*}\right)$ in which case we say that $A$ is a left-multiplier of $B$ (or $B$ is a right-multiplier of $A$ ), then the product $A \cdot B \equiv \overline{A(B\lceil\mathscr{D})}$ is a well defined element of 5 .

- If $A$ and $B$ satisfy only the weaker condition that $B \mathscr{D} \subseteq D\left(A^{* *}\right)$ and $A^{*} \mathscr{D} \subseteq D\left(B^{*}\right)$ another product $A \square B \equiv \overline{A^{\neq *}(B\lceil\mathscr{D})}$ may be defined.

In order to distinguish between the two partial products, we will denote the corresponding structures by $\mathbb{C}^{s}$ and $\mathbb{C}^{w}$ respectively.

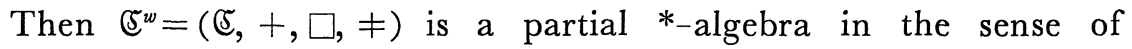
[2]: the three operations (sum, involution, partial product) satisfy the usual properties of distributivity between sum and product $(A \square(B \hat{+} \lambda C))=(A \square B) \hat{+} \lambda(A \square C), \lambda \in C$ and the usual relation between involution and product $(A \square B)^{*}=B^{*} \square A^{*}$ is verified.

So, $\widetilde{\mho}^{w}$ and all its vector subspaces, stable under $\square$ and containing 1 , are partial *-algebras.

In contrast to $\mathfrak{C}^{w}, \mathbb{C}^{s} \equiv(\mathfrak{S}, \hat{\mathcal{f}}, \cdot, \neq)$ is not a partial ${ }^{*}$-algebra. Although the sum, involution and partial product $\cdot$ are defined, examples are known [26], [27] where distributivity is violated: there exist operators $A, B$ and $T$ such that $T$ is a left multiplier of $A$ and $B$ but is not a left multiplier of $A \hat{+} B$ (the reason is that $D(A \hat{+} B)$ need not contain $D(A) \cap D(B))$. So, $\mathbb{f}^{s}$ itself is not a partial *-algebra but we may restrict ourselves to vector subspaces of ${ }^{5 s}$, containing 1 , stable under $\cdot$ and satisfying the distributivity law. We get in this way another class of partial $*_{\text {-algebras. }}$

A more restrictive class, the Op*-algebras, is obtained when every operator in $\mathfrak{A}$ maps $\mathscr{D}$ into itself i.e. $A \uparrow_{\mathscr{D}} \in L^{+}(\mathscr{D})$, the set of all 
closable operators in $\mathscr{H}$ such that $A \mathscr{D} \subseteq \mathscr{D}$ and $A^{*} \mathscr{D} \subseteq \mathscr{D}$.

For two such operators $A, B$, the product is always defined and coincides with the two partial products introduced above: $A B=A \cdot B$ $=A \square B=\overline{A(B\lceil\mathscr{D})}$.

An Op*-algebra is a ${ }^{*}$-subalgebra of $L^{+}(\mathscr{D})$ containing the unit operator.

\subsection{Domain considerations}

Given a $\neq$-invariant set $\mathfrak{A}$ on the domain $\mathscr{D}$, the corresponding graph-topology on $\mathscr{D}$ is given by the set of norms:

$\|f\|_{A}^{2}=\|f\|^{2}+\|A f\|^{2} \quad f \in \mathscr{D}$, where $A$ runs over $\mathfrak{A}$.

Every $A \in \mathfrak{A}$ is continuous from $\mathscr{D}$ with this topology into $\mathscr{H}$ with the norm topology.

Moreover, since $A$ is a closed operator, its domain $D(A)$ with the graph-norm is a Hilbert space that we shall denote by $\mathscr{H}_{A}$. The graph-topology on $\mathscr{D}$, (denote it $t_{\text {थ }}$ ) is then also the projective limit topology corresponding to the Hilbertian topologies of the $\mathscr{H}_{A}$ 's, $A \in \mathfrak{A}$.

The completion $\overline{\mathscr{D}}$ of $\mathscr{D}$ with respect to this projective topology is a closed subspace of $\mathscr{D}(\mathscr{U}) \equiv \bigcap_{A \in \mathscr{U}} \mathscr{H}_{A}$. A priori, this subspace might be a proper one contrary to the case of Op*-algebras where $\overline{\mathscr{D}}=\mathscr{D}(\mathfrak{H})$ [6] [28]. In fact, a sufficient condition for this equality is that the system of norms defining the topology of $\mathscr{D}$, be directed (i.e. $\forall A, B \in \mathfrak{A}$, there exists $C \in \mathfrak{U}$ such that $\left.\|f\|_{A},\|f\|_{B} \leq\|f\|_{C}, \forall f \in \mathscr{D}\right)$.

This happens in particular for Op*-algebras but need not hold for a general partial $*$-algebra or $\neq$-invariant set.

Although no explicit counterexample is known so far, we have to distinguish in the general case between $\mathscr{\mathscr { D }}$ and $\mathscr{D}(\mathfrak{C})$ i.e. we have $\mathscr{D} \subseteq \overline{\mathscr{D}} \subseteq \mathscr{D}(\mathfrak{H})=\underset{A \in \mathscr{T}}{\cap} \mathscr{H}_{A}$.

However [4] [6], we may always assume that $\mathscr{D}=\overline{\mathscr{D}}$, otherwise, we replace $\mathfrak{A}$ by its "closure" $\overline{\mathfrak{A}}$ which consists in the same set of closed operators as $\mathfrak{A}$ but considered as the closure of their restriction to $\overline{\mathscr{D}} \cdot \overline{\mathfrak{A}}$ is an extension of $\mathfrak{A}[15]$ (in particular $\overline{\mathfrak{A}}$ is isomorphic to $\mathfrak{A}$ i.e. all the algebraic operations are preserved).

Remark. It is also possible to consider an element of $\mathscr{A}$ as the closure of its restriction to $\mathscr{D}(\mathfrak{H})$, and by doing this, to get a $\neq$-invariant set or a partial $*$-algebra on $\mathscr{D}(\mathfrak{R})$. 
However, as shown in [3], it may happen that the new $\neq-$-invariant set be only homomorphic and not isomorphic to the initial set (for instance if $\mathscr{U} \subset \mathfrak{C}^{s}(\mathscr{D})$, the new set will in general be in $\mathfrak{S}^{w}(\mathscr{D}(\mathfrak{H}))$ i.e. the product is not preserved). In that case, we don't have extension in the sense of [15].

\subsection{Commutants}

Since we are dealing with unbounded operators, it is natural to consider also unbounded commutants. The biggest commutant we may introduce for a $\neq-$-invariant set $\mathfrak{X} \subseteq \mathfrak{C}$ is the "weak unbounded commutant":

$$
\mathfrak{V}_{\sigma}^{\prime}=\left\{X \in \mathfrak{S}:(X f, A g)=\left(A^{\ddagger} f, X^{\ddagger} g\right), \forall f, g \in \mathscr{D}, \forall A \in \mathfrak{X}\right\} .
$$

Some other unbounded commutants have been introduced in [30] [24] [25] [2] [3], all of them subsets of this one.

However, as far as we are concerned with the problem of irreducibility of a set of operators, it appears [6] [12] that only the bounded part of this commutant is relevant i.e. the "weak bounded commutant"

$\mathfrak{U}_{w}^{\prime}=\mathfrak{U}_{\sigma}^{\prime} \cap B(\mathscr{H}) \quad($ where $B(\mathscr{H})$ denotes the set of bounded opertors in $\mathscr{H})$.

A $\neq$-invariant set $\mathfrak{A}$ will be called irreducible iff $\mathfrak{U}_{w}^{\prime}$ consists in the scalar multiple identity only.

Since our operators are defined on some specified domain $\mathscr{D}$, a special role will also be played by the "strong bounded commutant" i.e. the elements of $\mathfrak{A}_{w}^{\prime}$ leaving $\mathscr{D}$ invariant.

$$
\begin{aligned}
\mathfrak{X}_{s}^{\prime}=\{ & X \in B(\mathscr{H}): X \mathscr{D} \subseteq \mathscr{D} \text { and } X^{*} \mathscr{D} \subseteq \mathscr{D}, X A f=A X f, \\
& \forall f \in \mathscr{D}, \forall A \in \mathscr{U}\} .
\end{aligned}
$$

(Rem: this $\mathfrak{I}_{s}^{\prime}$ is the ${ }^{*}$-invariant part of the $\mathfrak{U}_{s}^{\prime}$ introduced in [6] [15] [17].)

It is easy to check the elementary properties of those commutants [6] [15] [17]. We summarize them in three lemmas:

Lemma 1. 3.1. a) $\mathfrak{V}_{w}^{\prime}$ is a weakly closed linear subspace of $B(\mathscr{H})$ containing 1 and $*_{\text {-invariant. }}$

b) $\mathfrak{Y}_{s}^{\prime}$ is an algebra, *-invariant but not necessarily weakly closed.

c) $\overline{\mathfrak{U}_{s}^{\prime}}$ is weakly closed.

d) $\mathfrak{U}_{w}^{\prime}=\overline{\mathfrak{A}}_{w}^{\prime}$. 


\section{e) $\mathfrak{Y}_{s}^{\prime} \subseteq \overline{\mathfrak{Y}}_{s}^{\prime}$.}

Lemma 1.3.2. If $X \in \overline{\mathfrak{Y}}_{s}^{\prime}$ then $X$ is a bounded operator in the Hilbert space $\mathscr{H}_{A}$, for every $A \in \mathfrak{R}$. (Same proof as [17] Lemma 2.2.1. We use the fact that $\mathscr{D}$ is a core for any $A \in \mathfrak{U}_{\text {。 }}$ )

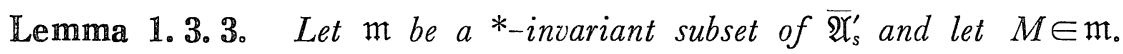
For every $A \in \mathfrak{A}, M$ leaves $D\left(A^{*}\right)$ invariant and $M$ commutes with $A^{*}$ (i.e. $\left.M A^{*} f=A^{*} M f \forall f \in D\left(A^{*}\right)\right)$. In particular, $M$ commutes with the self-adjoint operators $\left(1+A^{*} A\right)^{ \pm 1}, \quad\left(1+A^{*} A\right)^{ \pm 1 / 2}$ for any $A \in \mathfrak{U}$. (Same proof as [17] Lemma 2.2.2.)

Remark. $A^{*}$ is not a minimal operator. $\mathscr{D}$ is not necessarily a core for $A^{*}$. Nevertheless, $A^{*}$ is a closed operator and its domain $D\left(A^{*}\right)$ provided with the graph-norm is a Hilbert space $\mathscr{H}_{A^{*}}$

Lemma 1.3.4. Let $\mathfrak{m}$ be a von Neumann algebra contained in $\overline{\mathfrak{X}}_{s}^{\prime}$ and denote by $\mathfrak{m}_{A}$ its restriction to $\mathscr{H}_{A}$, for any $A \in \mathfrak{A}^{\text {. The family }}$ $\left\{\mathfrak{m}_{A} \mid A \in \mathfrak{X}\right\} \quad$ is a set of unitarily equivalent representations of $\mathfrak{m}$ in each $\mathscr{H}_{A}$ respectively. This equivalence is given by the unitary operators $\left\{\left(1+A^{*} A\right)^{ \pm 1 / 2}\right\}$ (between $\mathscr{H}$ and $\mathscr{H}_{A}$ ).

The proof follows from Lemmas 1.3.2. and 1.3.3. and the fact that the graph-norm in $\mathscr{H}_{A}$ may be rewritten as

$$
\|f\|_{A}^{2}=\|f\|^{2}+\|A f\|^{2}=\left\|\left(1+A^{*} A\right)^{1 / 2} f\right\|^{2} \text {. }
$$

\section{$\S$ II. Integral Decomposition of a Closed Operator}

\subsection{Decomposition of $\mathscr{H}$}

The method of integral decomposition we consider here is the one introduced in [17]. It consists in the identification of an unbounded closed operator $A \in \mathfrak{Q}$ with a bounded operator between $\mathscr{H}_{A}$ and $\mathscr{H}$. We then use the reduction theory for bounded operators but between different spaces. More explicitly, beginning with a $\neq-$-invariant set $\mathfrak{U}$, we shall consider an Abelian von Neumann algebra $\mathfrak{m} \subseteq \mathfrak{V}_{s}^{\prime}$ containing 1 and maximal in the sense that $\mathfrak{m}=\mathfrak{m}^{\prime} \cap \mathfrak{Y}_{w}^{\prime}\left(\mathfrak{H}^{\prime}\right.$ denotes the usual commutant for bounded operators).

By the extension theory developed in [15], we know that any $\neq-$ invariant set $\mathfrak{U}$ defined on $\mathscr{D}$ always admits an extension $\hat{\mathfrak{X}}$ on a 
domain $\hat{\mathscr{D}}$ dense in a larger Hilbert space $\hat{\mathscr{H}}$ for which such an $\mathfrak{m}$ exists. So, it is actually this extension $\hat{\mathfrak{U}}$ that we are going to decompose and, moreover, we may also assume $\hat{\mathfrak{U}}$ to be closed i.e. $\hat{\mathscr{D}}$ complete with respect to the graph topology (because of Lemma 1. 3. $1, \mathfrak{m}$ is maximal with respect to $\overline{\hat{\mathfrak{U}}}$ as well as with respect to $\hat{\mathfrak{U}}$ ).

Let thus $\mathfrak{U}$ be a closed $\neq$-invariant set and $\mathfrak{m}$ an Abelian von Neumann algebra such that $\mathfrak{m} \subseteq \mathfrak{U}_{s}^{\prime}$ and $\mathfrak{m}=\mathfrak{m}^{\prime} \cap \mathfrak{U}_{w}^{\prime}$. We may decompose the Hilbert space $\mathscr{H}$ in a direct integral on the spectrum of $\mathfrak{m}$ by the usual reduction theory for von Neumann algebras. More explicitly, there exists [13] a compact metrizable space $\Lambda$, a positive regular Borel measure $\mu$ on $\Lambda$ and a $\mu$-measurable field $\lambda \rightarrow \mathscr{H}(\lambda)$ of Hilbert spaces such that

$$
\mathscr{H} \simeq \int_{\Lambda} \mathscr{H}(\lambda) d \mu(\lambda)
$$

$\mathfrak{m}$ consists exactly in the diagonalizable operators in that decomposition i.e. $\mathfrak{m} \simeq L^{\infty}(\Lambda, \mu)$ and the set of bounded decomposable operators in $\mathscr{H}$ is exactly $\mathfrak{m}^{\prime} .[13]$

\section{2. Decomposition of $\mathscr{H}_{A}$}

Take now some $A \in \mathfrak{X}$. Between $\mathscr{H}$ and $\mathscr{H}_{A}$ we have the unitary operator $\left(1+A^{*} A\right)^{-1 / 2}$ (Lemma 1.3.4). Considered as operator in $\mathscr{H}$, this is a bounded operator commuting with every $M \in \mathfrak{m}$. Hence $\left(1+A^{*} A\right)^{-1 / 2}$ belongs to $\mathfrak{m}^{\prime}$ and is thus decomposable. There exists a $\mu$-measurable field $\lambda \rightarrow\left(1+A^{*} A\right)^{-1 / 2}(\lambda) \in B(\mathscr{H}(\lambda))$ essentially bounded such that:

$$
\left(1+A^{*} A\right)^{-1 / 2}=\int_{\Lambda}\left(1+A^{*} A\right)^{-1 / 2}(\lambda) d \mu(\lambda)
$$

and $\left\|\left(1+A^{*} A\right)^{-1 / 2}\right\|=$ ess. $\sup _{\lambda \in \Lambda}\left\|\left(1+A^{*} A\right)^{-1 / 2}(\lambda)\right\|$

Since $\left(1+A^{*} A\right)^{-1 / 2}(\lambda) \in B(\mathscr{H}(\lambda))$ for almost every $\lambda \in \Lambda$ (i.e. $\forall \lambda \in$ $\Lambda \backslash \mathscr{N}_{A}$ where $\mathscr{N}_{A}$ is a null-set) we may define:

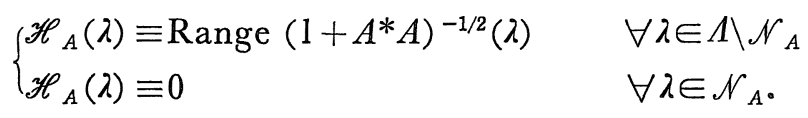

Defined in this way, $\mathscr{H}_{A}(\lambda)$ appears as a subset of $\mathscr{H}(\lambda)$ and it may be equipped with the following scalar product: If $f_{i}(\lambda)=\left(1+A^{*} A\right)^{-1 / 2}(\lambda) \mathrm{g}_{i}(\lambda)$, 
$i=1,2$ and $g_{i}(\lambda) \in \mathscr{H}(\lambda)$, put $\left(f_{1}(\lambda), f_{2}(\lambda)\right)_{A, \lambda} \equiv\left(g_{1}(\lambda), g_{2}(\lambda)\right)$.

With those definitions, the field $\lambda \rightarrow\left(1+A^{*} A\right)^{-1 / 2}(\lambda)$ is a field of unitary isomorphism between $\mathscr{H}(\lambda)$ and $\mathscr{H}_{A}(\lambda)$ for almost every $\lambda_{\text {。 }}$

Therefore [13], the inverses field $\lambda \leadsto\left[\left(1+A^{*} A\right)^{-1 / 2}(\lambda)\right]^{-1}$ is also measurable and defines the integral decomposition of $\left(1+A^{*} A\right)^{1 / 2}$ which is unbounded in $\mathscr{H}$ but unitary from $\mathscr{H}_{A}$ into $\mathscr{H}$.

Redefining $\left(1+A^{*} A\right)^{1 / 2}(\lambda)=\left[\left(1+A^{*} A\right)^{-1 / 2}(\lambda)\right]^{-1}$, we can rewrite the norm in $\mathscr{H}_{A}(\lambda)$ as:

$$
\|f(\lambda)\|_{A, \lambda}=\left\|\left(1+A^{*} A\right)^{1 / 2}(\lambda) f(\lambda)\right\|
$$

(for more details, see [17]). Of course, as expected, the field $\lambda \rightarrow \mathscr{H}_{A}(\lambda)$ will give the integral decomposition of $\mathscr{H}_{A}$ and corresponds to the decomposition of $\mathscr{H}_{A}$ with respect to the Abelian von Neumann algebra $\mathfrak{m}_{A}$ (which is unitarily equivalent to $\mathfrak{m}$ ).

In conclusion, we are able to decompose all the Hilbert spaces $\mathscr{H}_{A}$ on the same spectrum $\Lambda$ and with the same Borel measure $\mu$.

Moreover, this decomposition is coherent in the sense that, if $f \in \mathscr{H}_{A} \cap \mathscr{H}_{B}, f$ will admit a unique integral decomposition

$$
f=\int_{\Lambda} f(\lambda) d \mu(\lambda) \text { with } f(\lambda) \in \mathscr{H}_{A}(\lambda) \cap \mathscr{H}_{B}(\lambda)
$$

$\forall \lambda \in \Lambda \backslash\left(\mathscr{N}_{A} \cup \mathscr{N}_{B}\right)$ i.e. almost everywhere. Differences will only appear in expressions of the norms $\|f(\lambda)\|_{A, \lambda}$ and $\|f(\lambda)\|_{B . \lambda}$ as given above.

\section{3. Decomposition of a single operator}

As we said above, when $\mathscr{H}$ is decomposed in a direct integral on the spectrum $\Lambda$ of $\mathfrak{m}$, the set of ail bounded decomposable operators is exactly $\mathfrak{m}^{\prime}$.

Here we shall use a slightly more general result [13].

Let $\mathscr{H}$ and $\mathscr{H}^{\prime}$ be two Hilbert spaces decomposed on the same $\Lambda$ with respect to the same measure $\mu_{\text {。 }}$ Every function $m \in L^{\infty}(\Lambda, \mu)$ determines a diagonal operator $M$ in $\mathscr{H}$ and another one $M^{\prime}$ in $\mathscr{H}^{\prime}$. In that situation, every bounded operator from $\mathscr{H}$ into $\mathscr{H}^{\prime}$ which intertwines $M$ and $M^{\prime}$ (for any $m \in L^{\infty}(\Lambda, \mu)$ ), is decomposable.

In our case, every $A \in \mathfrak{A}$ is a bounded operator from its domain $\mathscr{H}_{A}$ into $\mathscr{H}$ and we have decomposed $\mathscr{H}$ and $\mathscr{H}_{A}$ in a direct integral on the spectrum of $\mathfrak{m}$. Any $m \in L^{\infty}(\Lambda, \mu)$ defines an operator $M \in \mathfrak{m}$ 
$\subseteq B(\mathscr{H})$ and $M_{A} \equiv M \uparrow \mathscr{H}_{A} \in \mathfrak{H}_{A} \subseteq B\left(\mathscr{H}_{A}\right)$ (remember Lemmas 1.3.21.3.4). Moreover, since $\mathfrak{m} \subseteq \mathfrak{U}_{s}^{\prime}$, we have $M A f=A M_{A} f, \forall f \in \mathscr{D}$, i.e. $A$ intertwines $M$ and $M_{A}$. Hence $A$ is decomposable in the sense that there exists a measurable field $\lambda \rightarrow A(\lambda) \in B\left(\mathscr{H}_{A}(\lambda), \mathscr{H}(\lambda)\right)$, essentially bounded, such that:

$$
A=\int_{\Lambda} A(\lambda) d \mu(\lambda)
$$

and $\|A\|_{B\left(\mathscr{H}_{A}, \mathscr{H}\right)}=$ ess. $\sup _{\lambda \in \Lambda}\|A(\lambda)\|_{B\left(\mathscr{H}_{A}(\lambda), \mathscr{H}(\lambda)\right) \cdot}$

If we consider $A(\lambda)$ as an operator in $\mathscr{H}(\lambda)$ with domain $\mathscr{H}_{A}(\lambda)$, we get a closed operator in $\mathscr{H}(\lambda)$. So, the closed operator $A$ in $\mathscr{H}$ is decomposed in a direct integral of closed operators $A(\lambda)$ in $\mathscr{H}(\lambda)$ (for almost every $\lambda \in \Lambda$ i.e. $\lambda \in \Lambda \backslash \mathscr{N}_{A}^{\prime}$, where $\mathscr{N}_{A}^{\prime}$ is a null-set containing the previous null-set $\mathscr{N}_{A}$ ).

Since $\mathscr{H}_{A}(\lambda)$, which was already a Hilbert space with its own scalar product, is now also the domain of the closed operator $A(\lambda)$, the norm $\|f(\lambda)\|_{A, \lambda}$ introduced before, is equivalent to the graph-norm

$$
\|f(\lambda)\|_{A(\lambda)}^{2}=\|f(\lambda)\|^{2}+\|A(\lambda) f(\lambda)\|^{2} \text { 。 }
$$

It may happen that $A$ is also a bounded operator from some $\mathscr{H}_{B}$ in some $\mathscr{H}_{C}(B, C \in \mathfrak{U})$. This happens for instance if $\mathfrak{U}$ is an Op*algebra because then $A$ is continuous from $\mathscr{D}$ to $\mathscr{D}$ with the projective topology. Hence, for any $C \in \mathfrak{U}$, there exists $B \in \mathfrak{U}$ such that $\|A f\|_{C} \leq$ $K\|f\|_{B}, \forall f \in \mathscr{D}$ and some constant $K$. This means that $A$ may be extended to a bounded operator $A_{C B}$ from $\mathscr{H}_{B}$ into $\mathscr{H}_{C}$ (in fact $A_{C B}=A\left(\mathscr{H}_{B}\right)$.

Here again, every $m \in L^{\infty}(\Lambda, \mu)$ determines a diagonal operator $M_{B}$ in $\mathscr{H}_{B}$ and a diagonal operator $M_{C}$ in $\mathscr{H}_{C}$ and $A$ intertwines those two operators. Hence $A$ is decomposable and we get a measurable field $\lambda \rightarrow A_{C B}(\lambda) \in B\left(\mathscr{H}_{B}(\lambda), \mathscr{H}_{C}(\lambda)\right)$.

Each time $A$ has some boundedness properties between any two Hilbert spaces, the same boundedness properties hold at (almost) every $\lambda$-level of the integral decomposition.

All the decompositions we may obtain in this way (between different pairs of Hilbert spaces) are coherent in the sense that they are all restrictions of the first one between $\mathscr{H}_{A}=D(A)$ and $\mathscr{H}$ : $A_{C_{B}}(\lambda)=A(\lambda) \uparrow \mathscr{H}_{B}(\lambda)$ 


\subsection{Which are the decomposable operators?}

We have been able to decompose an element $A \in \mathfrak{A}$ for three reasons:

a) $\mathscr{H}_{A}$ and $\mathscr{H}$ are both decomposed on the same $\Lambda=$ Sp m.

b) $A$ is a bounded operator from $\mathscr{H}_{A}$ into $\mathscr{H}$.

c) $A$ intertwines $\mathfrak{m}$ and $\mathfrak{m}_{A}$.

Obviously, elements of $\mathscr{U}$ are certainly not the only minimal closed operators satisfying those conditions.

Theorem. Every $X \in \mathfrak{H}_{\sigma}^{\prime}$ is decomposable.

Proof. By definition, $\mathfrak{m}_{\sigma}^{\prime}=\left\{X \in \mathfrak{C}:\left(X^{ \pm} f, M g\right)=\left(M^{ \pm} f, X g\right), \forall f, g \in \mathscr{D}\right.$, $\forall M \in \mathfrak{m}\}$. Using the facts that elements of $\mathfrak{m}$ are bounded and leave $\mathscr{D}$ invariant $\left(\mathfrak{m} \subseteq \mathfrak{X}_{s}^{\prime}\right.$ ) we have $M X g=X^{* *} M g=X M g, \forall g \in \mathscr{D}, \forall M \in \mathfrak{m}$. Since $\mathscr{D}$ is a core for $X$ and $M$ is bounded in $\mathscr{H}$, the equality $M X g=X M g$ extends from $\mathscr{D}$ to $\mathscr{H}_{X}=D(X)$. From this, it also follows that $M$ is bounded in $\mathscr{H}_{X}:\|M g\|_{X}^{2}=\|M g\|^{2}+\|X M g\|^{2}=\|M g\|^{2}+\|M X g\|^{2} \leq$ $\|M\|^{2} \|\left. g_{1}^{2}\right|_{X}, \forall g \in \mathscr{H}_{X}$.

Finally, $\mathfrak{m}_{X}$, the restriction of $\mathfrak{m}$ to $\mathscr{H}_{X}$, is an Abelian von Neumann algebra unitarily equivalent to $\mathfrak{m}$ in $\mathscr{H}$ (by the operator $\left(1+X^{*} X\right)^{ \pm 1 / 2}$ and $\mathscr{H}$ and $\mathscr{H}_{X}$ are both decomposable in a direct integral on $\mathrm{Sp} \mathrm{m}$.

The three conditions mentioned above are fulfilled, which means that $X$ is decomposable in a direct integral of closed operator $X(\lambda)$ in $\mathscr{H}(\lambda)$ respectively.

Remark. Since we had $\mathfrak{m} \subseteq \mathfrak{A}_{s}^{\prime} \subseteq \mathfrak{Y}_{\sigma}^{\prime}$, we get also $\mathfrak{A} \subseteq \mathfrak{Y}_{\sigma o}^{\prime \prime} \subseteq \mathfrak{m}_{\sigma}^{\prime}$ and this explains why the elements of $\mathfrak{A}$ are decomposable. We have just shown now that they are not the only ones. Moreover, Lemma 1.3.3 suggests another class of decomposable operators, namely, adjoints of elements of $\mathfrak{m}_{\sigma}^{\prime}$. They are no longer minimal operators but maximal ones [2] and $\mathscr{D}$ is no longer a core for them. Nevertheless, they are closed operators and their domains are Hilbert spaces.

Theorem. If $X \in \mathfrak{m}_{o}^{\prime}, X^{*}$ is decomposable.

Proof: For $X \in \mathfrak{m}_{\sigma}^{\prime}$ we have seen in the proof of the last theorem 
that $M X g=X M g$ for every $g \in D(X)$ and for every $M \in \mathfrak{m}$. Take in particular $M^{*} \in \mathfrak{m}$ and consider $h \in D\left(X^{*}\right)$. We compute

$$
(M h, X g)=\left(h, M^{*} X g\right)=\left(h, X M^{*} g\right)=\left(X^{*} h, M^{*} g\right) \text {. }
$$

Since $M^{*}$ is bounded, this means that $M h \in D\left(X^{*}\right)$ and $X^{*} M h$ $=M X^{*} h, \forall h \in D\left(X^{*}\right)$. So, $M$ commutes with $X^{*}$ and the same calculation as in the previous theorem shows that $M$ is bounded on $\mathscr{H}_{X *}$.

Conclusion. In this section, we gave a method of decomposition of a closed operator into a direct integral of closed operators. We have seen that if $X$ possesses some boundedness properties, the same properties will hold at the $\lambda$-level for almost every $\lambda \in \Lambda$. We did not say anything so far about the minimality or maximality of the $X(\lambda)$ so obtained. To do so, we have to specify some domain $\mathscr{D}(\lambda)$ dense in $\mathscr{H}(\lambda)$ which will be (or not be) a core for $X(\lambda)$. We shall consider this problem in the next section in the case of a $\neq$-invariant family $\mathfrak{U}$. We consider first a countable family of minimal operators and we decompose them simultaneously. The countability assumption is necessary to avoid problems of null-sets. The extension to uncountable families will be done in Part II. Once we have a common dense domain $\mathscr{D}(\lambda)$ on which all $A(\lambda)$ 's are defined, we may also look at the continuity properties of $A(\lambda)$ with respect to some graphtopology.

\section{§III. A Dense Domain $\mathscr{D}(\lambda)$ for a Countable Set of Operators-Metrizability Assumption}

3. 1. In this paragraph, $\mathfrak{A}$ will denote a countable (closed) $\neq$-invariant set. Applying the method of decomposition just explained to every element $A \in \mathfrak{A}$, we get closed operators $A(\lambda)$ in $\mathscr{H}(\lambda)$ for $\lambda \in \Lambda \backslash \mathscr{N}_{A}^{\prime}$. Thus, it is only for $\lambda \in \Lambda \backslash \cup_{A \in \mathscr{N}} \mathscr{N}_{A}^{\prime}$ that all the $A(\lambda)$ 's may simultaneously be considered as a set of closed operators in the Hilbert space $\mathscr{H}(\lambda)$. The assumption that $\mathfrak{A}$ be countable guarantees that the set of exceptional $\lambda$ 's to disregard is still a null-set.

Under that assumption, we are now able to work at the $\lambda$-level (for almost every $\lambda \in \Lambda$ ) and to ask the next question: 
Can we find a dense domain $\mathscr{D}(\lambda)$ of $\mathscr{H}(\lambda)$ which will be a common domain for all $A(\lambda)$ 's ?

3. 2. If $\mathfrak{A}$ is countable, $\mathscr{D}(=\overline{\mathscr{D}})$ provided with the graph-topology $t_{\mathfrak{A}}$ given by the norms $\left\{\|\cdot\|_{A}: A \in \mathfrak{Q}\right\}$ is a Fréchet space.

However, as we mentioned already, it might be a proper closed subspace of $\mathscr{D}(\mathfrak{U})=\bigcap_{A \in \mathfrak{A}} \mathscr{H}_{A}$.

In other words and following the terminology of [2], $\mathfrak{X}$ is closed but not necessarily "fully closed".

An important consequence of the fact that $\mathscr{D}$ is a Fréchet space is that every element $X \in \mathscr{C}(\mathscr{D})$ will be continuous from $\mathscr{D}$ into $\mathscr{H}$. Indeed, $X$ being a closed operator in $\mathscr{H}$ is also closed from $\mathscr{D}$ to $\mathscr{H}$ and hence is continuous by the closed graph theorem. In particular, the topology $t_{\mathbb{E}(\mathscr{D})}$ is equivalent to $t_{\mathscr{U}}$ on $\mathscr{D}$.

We can now prove the following result:

Theorem. There exists a countable set $\mathscr{D}_{0}=\left\{e_{1}, e_{2}, \ldots\right\}$ dense in $\mathscr{D}$ for the graph-topology $t_{\mathfrak{I}}$ such that for almost every $\lambda \in \Lambda, \mathscr{D}_{0}(\lambda) \equiv\left\{e_{1}(\lambda)\right.$, $\left.e_{2}(\lambda), \ldots \mid e_{i} \in \mathscr{D}_{0}\right\}$ is a core for $A(\lambda)$, for any $A \in \mathfrak{U}$.

Proof. a) In the explicit construction of a direct integral of Hilbert spaces

$$
\mathscr{H}=\int_{\Lambda} \mathscr{H}(\lambda) d \mu(\lambda)
$$

on the spectrum of a von Neumann algebra, there always exists a countable dense set $\left\{x_{1}, x_{2}, \ldots\right\} \subseteq \mathscr{H}$ such that the set of images $\left\{x_{1}(\lambda), x_{2}(\lambda), \ldots\right\}$ is dense in $\mathscr{H}(\lambda)$ for almost every $\lambda \in \Lambda$ (see Dixmier p. 208). Since $\mathscr{D}$ is dense in $\mathscr{H}$, we may always choose this set $\left\{x_{i}\right\}$ contained in $\mathscr{D}$.

Similarly, in the decomposition of $\mathscr{H}_{A}$ in direct integral, we may find another set $\left\{x_{i, A}\right\}$ dense in $\mathscr{H}_{A}$ such that $\left\{x_{i, A}(\lambda)\right\}$ is dense in $\mathscr{H}_{A}(\lambda)$. Since $\mathscr{D}$ is dense in $\mathscr{H}_{A}$, for the $A$-graph-topology, $\left\{x_{i, A}\right\}$ may be chosen in $\mathscr{D}$. We can do the same thing for every $A \in \mathfrak{U}$, but the set $\left\{x_{i, A}\right\}$ may be different for each $A$. However, those dense sets will be useful later.

b) Since $\mathscr{D}$ is a separable Fréchet space, there exists a countable 
set $\mathscr{D}_{0}=\left\{e_{1}, e_{2}, e_{3}, \ldots\right\}$ everywhere dense in $\mathscr{D}$ for the Fréchet topology $t_{\mathfrak{r}}$. So, any $f \in \mathscr{D}$ is a $t_{\mathfrak{2}}$-limit of a sequence $\left\{f_{i}\right\}$ of elements of $\mathscr{D}_{0}$ i.e., for any $A \in \mathfrak{U}$ we have ||$f-f_{i} \|_{A} \rightarrow 0$ (and the sequence $\left\{f_{i}\right\}$ is independent of $A$ ). Decomposing $f$ and $f_{i}$ in a direct integral, we get:

$$
\left\|f-f_{i}\right\|_{A}^{2}=\int_{\Lambda}\left\|f(\lambda)-f_{i}(\lambda)\right\|_{A, \lambda}^{2} \quad d \mu(\lambda) .
$$

Since the 1.h.s. tends to zero, there exists a subsequence $\left\{f_{i_{k}}\right\}$ such that:

$$
\left\|f(\lambda)-f_{i_{k}}(\lambda)\right\|_{A, \lambda} \rightarrow 0 \text { in } \mathscr{H}_{A}(\lambda) \text { a.e. }
$$

Moreover, since there is only a countable number of different norms to consider, the subsequence $\left\{f_{i_{k}}\right\}$ may be chosen independant of $A$ i.e., the relation ( $*$ ) holds in every $\mathscr{H}_{A}(\lambda)$ (for almost every $\lambda \in \Lambda$ ).

Finally, $f(\lambda)$ belongs to the completion of $\mathscr{D}_{0}(\lambda)=\left\{e_{1}(\lambda), e_{2}(\lambda), \ldots\right.$ $\left.\mid e_{i} \in \mathscr{D}_{0}\right\}$ with respect to the projective topology defined by the graph-norms of all $A(\lambda)$ 's $(\forall A \in \mathfrak{U})$ :

$$
f(\lambda) \in \overline{\mathscr{D}_{0}(\lambda)} \text { (almost everywhere). }
$$

c) Now we show that $\mathscr{D}_{0}(\lambda)$ is a core for every $A(\lambda)$.

Consider some $g(\lambda) \in \mathscr{H}_{A}(\lambda)$. By the considerations of point a) of this proof, we know that there exists a sequence $\left\{g_{i}\right\} \subseteq \mathscr{D}$, dense in $\mathscr{H}_{A}$, such that $g(\lambda)$ is the limit of $g_{i}(\lambda)$ in the $A(\lambda)$-graph-topology. Since $g_{i} \in \mathscr{D}$, point b) tells us that, in the integral decomposition, we shall have for every $i: g_{i}(\lambda) \in \overline{\mathscr{D}_{0}(\lambda)}$. In turn, $g_{i}(\lambda)$ will be the limit of elements $g_{i, n}(\lambda) \in \mathscr{D}_{0}(\lambda)$, in the $A(\lambda)$-graph-topology. Hence $g(\lambda)=A(\lambda)-\lim _{i} g_{i}(\lambda)=A(\lambda)-\lim _{i, n} g_{i, n}(\lambda)=A(\lambda)-\lim _{j} g_{j j}(\lambda)$, by a diagonal procedure. Finally, $g(\lambda)$ is a limit of elements of $\mathscr{D}_{0}(\lambda)$ in the $A(\lambda)$-graph-topology hence $\mathscr{D}_{0}(\lambda)$ is dense in $\mathscr{H}_{A}(\lambda)$ and is a core for $A(\lambda)$.

\section{3. A common domain}

Theorem. Define $\mathscr{D}(\lambda) \equiv \overline{\mathscr{D}_{0}(\lambda)}$ as the completion of $\mathscr{D}_{0}(\lambda)$ with respect to the projective topology defined by all the $A(\lambda)$-graph-norms. Then $\forall A \in \mathfrak{A}, A(\lambda)$ is a $\mathscr{D}(\lambda)$-minimal operator (i.e. $A(\lambda) \in \mathfrak{S}(\mathscr{D}(\lambda)))$ and $A(\lambda)$ is continuous from $\mathscr{D}(\lambda)$ with this topology into $\mathscr{H}(\lambda)$ for almost 
every $\lambda \in \Lambda$.

Proof. We just saw that $\mathscr{D}_{0}(\lambda)$ hence a fortiori $\overline{\mathscr{D}_{0}(\lambda)}=\mathscr{D}(\lambda)$ is a core for any $A(\lambda)$. Thus $A(\lambda)=\overline{A(\lambda) \Gamma_{\mathscr{D}_{0}(\lambda)}}=\overline{A(\lambda) \Upsilon_{\mathscr{D}(\lambda)}}$ is a minimal operator with respect to $\mathscr{D}(\lambda)$. Since $\mathscr{D}(\lambda)$ is by construction complete in the projective topology, we get that $\{A(\lambda) \mid A \in \mathfrak{Q}\}$ is a closed set of $\mathscr{D}(\lambda)$-minimal operators in $\mathscr{H}(\lambda)$.

Moreover, the fact that $A$ is continuous from $\mathscr{D}$ (with $t_{\mathfrak{2}}$ ) to $\mathscr{H}$ which is expressed by the inequality:

$$
\|A f\|^{2} \leq K\|f\|_{A}^{2} \quad \forall f \in \mathscr{D}
$$

gives rise in the integral decomposition to inequalities of the form:

$$
\|A(\lambda) f(\lambda)\|^{2} \leq K\|f(\lambda)\|_{A, \lambda}^{2}
$$

for almost every $\lambda \in \Lambda$ and for every $f \in \mathscr{D}$. In particular, this is true for every $e_{i} \in \mathscr{D}_{0}$, hence $A(\lambda)$ is continuous from $\mathscr{D}_{0}(\lambda)$ with the projective topology into $\mathscr{H}(\lambda)$ and thus can be extended to $\mathscr{D}(\lambda)$ in a continuous operator.

Remarks. 1. The common domain $\mathscr{D}(\lambda)$ so defined is in general a proper closed subspace of $\underset{A \in \mathscr{A}}{\cap} \mathscr{H}_{A}(\lambda)$. However, $\mathscr{D}(\lambda)$ is large enough in the sense that it contains the images $f(\lambda)$ of all $f \in \mathscr{D}$ so that expressions of the type

$$
A f=\int_{\Lambda} A(\lambda) f(\lambda) d \mu(\lambda) \quad \text { make sense } \forall f \in \mathscr{D}, \forall A \in \mathfrak{X} \text {. }
$$

2. In the case where the system of norms defining the topology of $\mathscr{D}$ is directed, in particular if $\mathscr{A}$ is an Op*-algebra, we have $\mathscr{D}=\bigcap_{A \in \mathfrak{U}} \mathscr{H}_{A}$. In the integral decomposition, the inequalities between norms are translated in analogous inequalities between the $A(\lambda)$ norms, for almost every $\lambda \in \Lambda$. The system of $A(\lambda)$-graph-norms is thus also directed and this implies that

$$
\mathscr{D}(\lambda)=\bigcap_{A \in \mathscr{\mathscr { A }}} \mathscr{H}_{A}(\lambda) \text {. }
$$

In particular, we recover the result of [17] where we were decomposing Op*-algebras only and where we had introduced the common domain immediately by this last equality. Moreover, in the case of Op*-algebras, the elements $A \in \mathfrak{U}$ leave the domain $\mathscr{D}$ invariant and 
are continuous from $\mathscr{D}$ into itself for the projective topology. Since inequalities between norms are preserved in the integral decomposition, the boundedness properties of $A$ are translated in boundedness properties of $A(\lambda)$. It follows that each $A(\lambda)$ is continuous from $\mathscr{D}(\lambda)$ into itself with the projective topology defined by the norms $\left\{\|\cdot\|_{A(\lambda)}: A \in \mathfrak{Q}\right\}$.

We shall now see that because $\mathscr{D}$ is metrizable and as a consequence of this every $X \in \mathfrak{C}(\mathscr{D})$ is continuous from $\left(\mathscr{D}, t_{\mathfrak{l}}\right)$ into $\mathscr{H}$, the results of the two previous theorems extend to all decomposable operators.

Theorem. Let $X \in \mathfrak{m}_{\sigma}^{\prime}$ (i.e. a decomposable operator). Then $\mathscr{D}_{0}(\lambda)$ and $\mathscr{D}(\lambda)$ are cores for $X(\lambda)$ and $X(\lambda)$ is continuous from $\left(\mathscr{D}(\lambda), t_{\mathscr{Q}(\lambda)}\right)$ into $\mathscr{H}(\lambda)$ for almost every $\lambda \in \Lambda$.

Proof. The fact that $X$ is continuous from $\left(\mathscr{D}, t_{\mathfrak{2}}\right)$ into $\mathscr{H}$ is expressed by an inequality of the type $\|X f\| \leq K\|A f\|, \forall f \in \mathscr{D}$, some constant $K$ and some $A \in \mathfrak{Y}$. In the integral decomposition, we get a similar inequality with $X(\lambda), f(\lambda), A(\lambda)$, in particular $\forall f \in \mathscr{D}_{0}$ which means that $X(\lambda)$ is continuous from $\left(\mathscr{D}_{0}(\lambda), t_{\mathscr{U}(\lambda)}\right)$ into $\mathscr{H}(\lambda)$ and may be extended in a continuous operator from $\left(\mathscr{D}(\lambda), t_{\mathscr{\Re ( \lambda )}}\right)$ into $\mathscr{H}(\lambda)$. As in the theorem of Section 3.2., any $g(\lambda) \in \mathscr{H}_{X}(\lambda)$ is the limit of a sequence $\left\{g_{i}(\lambda)\right\}$ with $g_{i} \in \mathscr{D}$. Hence each of those $g_{i}$ 's is a limit of elements of $\mathscr{D}_{0}$ such that $g_{i}(\lambda)$ is a limit of elements of $\mathscr{D}_{0}(\lambda)$ in the $\mathfrak{U}(\lambda)$-graph topology. But this implies that $g_{i}(\lambda)$ is also a limit of elements of $\mathscr{D}_{0}(\lambda)$ in the $X(\lambda)$-graph norm. Finally, this means that $\mathscr{D}_{0}(\lambda)$ is a core for $X(\lambda)$.

\section{§IV. Verification of the Algebraic Properties}

4. 1. Theorem. Let $\mathfrak{X}$ be a countable $\neq-$-invariant set of $\mathscr{D}$-minimal operators. Assume any $A \in \mathfrak{X}$ is decomposed in a direct integral following the method of Section II. The following algebraic relations hold for almost every $\lambda \in \Lambda$ and $\forall A, B \in \mathfrak{X}$.

a) $A(\lambda)^{\ddagger}=A^{\neq}(\lambda)$.

b) $(A \uparrow B)(\lambda)=A(\lambda) \uparrow B(\lambda)$. 
c) If $A . B$ is defined then $A(\lambda) . B(\lambda)$ is defined and $(A . B)(\lambda)=$ $A(\lambda) . B(\lambda)$.

d) $A^{*}(\lambda) \subset A(\lambda)^{*}$ and $A^{* *}(\lambda) \subset A(\lambda)^{* *}$.

e) If $A \square B$ is defined then $A(\lambda) \square B(\lambda)$ is defined and $(A \square B)(\lambda)=$ $A(\lambda) \square B(\lambda)$.

Remark. The same theorem holds if we replace in the hypotheses, $A, B \in \mathfrak{X}$ by $A, B \in \mathfrak{m}_{\sigma}^{\prime}$ i. e. any pair of decomposable operators. In fact, because $\mathfrak{m} \subseteq B(\mathscr{H})$ and $\mathfrak{m} \mathscr{D} \subseteq \mathscr{D}$, it can be shown that $\mathfrak{m}_{\sigma}^{\prime}$ is a

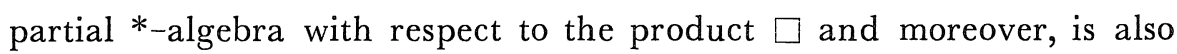
stable with respect to the product. [30]

\section{Proof. a) The adjoint}

Since $A(\lambda)$ is $\mathscr{D}(\lambda)$-minimal, the definition of $A(\lambda)^{\neq}$is of course $A(\lambda)^{\mp}=\overline{A(\lambda)^{*} \uparrow \mathscr{D}(\lambda) \cdot}$ Take $g \in D(A)$ and $f \in D\left(A^{\mp}\right)$. For every $M \in \mathfrak{m}$ we have $(f, M A g)=\left(A^{\neq} f, M g\right)$, which gives in the integral decomposition:

$$
\int_{\Lambda} m(\lambda)\left[(f(\lambda), A(\lambda) g(\lambda))-\left(A^{+}(\lambda) f(\lambda), g(\lambda)\right)\right] d \mu(\lambda)=0
$$

for every $m \in L^{\infty}(\Lambda, \mu)$.

This implies that for almost every $\lambda \in \Lambda$ :

$$
(f(\lambda), A(\lambda) g(\lambda))=\left(A^{\neq}(\lambda) f(\lambda), g(\lambda)\right) .
$$

This relation which holds for $g \in D(A)$ and $f \in D\left(A^{\ddagger}\right)$, extends by Theorem 3.2 to any $g(\lambda) \in D(A(\lambda))$ and any $f(\lambda) \in D\left(A^{*}(\lambda)\right)$. Hence, $D\left(A^{*}(\lambda)\right)=\mathscr{H}_{A^{*}}(\lambda) \subseteq D\left(A(\lambda)^{*}\right)$ and $A^{*}(\lambda)$ and $A(\lambda)^{*}$ coincide on $\mathscr{H}_{A^{\ddagger}}(\lambda)$ which contains the common domain $\mathscr{D}(\lambda)$. Since we know that $A^{\neq}(\lambda)$ is $\mathscr{D}(\lambda)$-minimal:

$$
A^{\ddagger}(\lambda)=\overline{A^{\ddagger}(\lambda) \Upsilon_{\mathscr{D}(\lambda)}}=\overline{A(\lambda)^{*} \Upsilon_{\mathscr{D}(\lambda)}}=A(\lambda)^{\ddagger},
$$

which proves the a) of the theorem.

\section{b) The sum}

Let $C \equiv A \hat{+} B=\overline{A \uparrow \mathscr{D}+B \Upsilon_{\mathscr{D}}}$. For any $g \in \mathscr{H}, f \in \mathscr{D}, M \in \mathfrak{m}$, we have $(g, C M f)=(g, A M f)+(g, B M f)$. Taking the integral decomposition as in a) above, we can easily derive that: $(g(\lambda), C(\lambda) f(\lambda))=(g(\lambda)$, $A(\lambda) f(\lambda)+(g(\lambda), B(\lambda) f(\lambda))$. Since the set of $g(\lambda)$, coming from some $g$ in $\mathscr{H}$ is dense in $\mathscr{H}(\lambda)$, we get: $C(\lambda) f(\lambda)=A(\lambda) f(\lambda)+B(\lambda) f(\lambda)$ for 
any $f \in \mathscr{D}$. This is true in particular for any $f \in \mathscr{D}_{0}$, the countable set dense in $\mathscr{D}$, that we have introduced in 3.2. Hence, $C(\lambda) \Upsilon_{\mathscr{D}_{0}(\lambda)}$ $=(A(\lambda)+B(\lambda)) \uparrow \mathscr{D}_{0}(\lambda)$. We have seen in 3.3 that $\mathscr{D}_{0}(\lambda)$ is a core for any $C(\lambda)$, because $G \in \mathfrak{m}_{\sigma}^{\prime}$. Hence, $\overline{C(\lambda) \Upsilon_{\mathscr{D}_{0}(\lambda)}}=C(\lambda)$. On the other hand, since $\mathscr{D}_{0}(\lambda)$ is by construction dense in $\mathscr{D}(\lambda)$ for the projective topology, it is a fortiori dense in $\mathscr{D}(\lambda)$ for the topology defined by the norm $\|\cdot\|_{A(\lambda)+B(\lambda)}$ which is dominated by $\|\cdot\|_{A(\lambda)}+\|\cdot\|_{B(\lambda)}$. (Remark: it does not follow from this that $\mathscr{D}_{0}(\lambda)$ is dense in $\mathscr{H}_{A}(\lambda) \cap \mathscr{H}_{B}(\lambda)$, counterexamples do exist [26]). It follows from this that $\mathscr{D}_{0}(\lambda)$ is also a core for $A(\lambda) \hat{f} B(\lambda)$ and that

$$
\overline{(A(\lambda)+B(\lambda)) \Upsilon_{\mathscr{D}_{0}(\lambda)}}=\overline{(A(\lambda)+B(\lambda)) ्_{\mathscr{D}(\lambda)}}=A(\lambda) \uparrow B(\lambda) .
$$

c) The product

Let $A, B \in \mathfrak{U}$ such that $A . B$ is defined (it then belongs to $\mathfrak{m}_{\sigma}^{\prime}$ ). By definition of the product ., this means that Range $(B \uparrow \mathscr{D}) \subseteq D(A)=\mathscr{H}_{A}$ and Range $\left(A^{\neq} \uparrow \mathscr{D}\right) \subseteq D\left(B^{\ddagger}\right)=\mathscr{H}_{B^{*}}$.

Since every element of $\mathfrak{m}_{\sigma}^{\prime}$ is continuous from $\mathscr{D}$ with the projective topology into $\mathscr{H}$, the fact that $A . B$ is defined means in particular that $B$ maps $\mathscr{D}$ continuously into $D(A)$ i. e. there exists a finite set of elements $C_{1}, C_{2}, \ldots C_{N}$ of $\mathfrak{X}$ such that: $\|B h\|_{A} \leq K \sum_{i=1}^{N}\|h\|_{C_{i}} \forall h \in \mathscr{D}$ i. e. $B$ maps continuously some closed subset of $\overbrace{i=1}^{N} \mathscr{H}_{C_{i}}{ }^{i=1}$ (the completion of $\mathscr{D}$ with respect to $\sum_{i=1}^{N}\|\cdot\|_{C_{i}}$ into $\mathscr{H}$. If we consider the integral decomposition of $B, h, A, G_{1}, \ldots C_{N}$, we get a similar inequality for almost every $\lambda \in \Lambda$ :

$$
\|B(\lambda) h(\lambda)\|_{A(\lambda)} \leq K \sum_{i=1}^{N}\|h(\lambda)\|_{C_{i}(\lambda)}
$$

which is true in particular for all $h \in \mathscr{D}_{0}$. Hence $B(\lambda)$ maps continuously $\mathscr{D}_{0}(\lambda)$ (and $\mathscr{D}(\lambda)$ by continuity) with the projective topology into $\mathscr{H}_{A}(\lambda)$. Finally, Range $\left(B(\lambda) \uparrow \mathscr{D}(\lambda) \subset \mathscr{H}_{A}(\lambda)=D(A(\lambda))\right.$. Similarly with the second condition, we get:

$$
\text { Range }\left(A^{\neq}(\lambda) \uparrow_{\mathscr{D}(\lambda)}\right) \subset \mathscr{H}_{B^{ \pm}}(\lambda)=D\left(B^{\neq}(\lambda)\right) .
$$

In conclusion, if $B$ is a right multiplier of $A, B(\lambda)$ will be a right multiplier of $A(\lambda)$ i. e. the product $A(\lambda) . B(\lambda)$ is defined almost everywhere. It remains to show that it is equal to $(A . B)(\lambda)$. As we did for the sum, it is easy to get that

$$
(A . B)(\lambda){\mathscr{D}_{0}(\lambda)}=A(\lambda) B(\lambda) \Upsilon_{\mathscr{D}_{0}(\lambda)}
$$


and the closure of the $\mathrm{l}_{\circ}$ h. S. is by definition $(A . B)(\lambda)$ because $A . B \in$ $\mathfrak{m}_{\sigma \circ}^{\prime} \quad$ On the other hand, since $\left(A_{\circ} B\right)(\lambda)$ is $\mathscr{D}(\lambda)$-minimal and since $\mathscr{D}(\lambda)$ is the completion of $\mathscr{D}_{0}(\lambda)$ with respect to $t_{\mathscr{A}(\lambda)}$ and a fortiori with respect to $t_{\widetilde{E}(\mathscr{D}(\lambda))}$ we have for any $f(\lambda) \in \mathscr{D}(\lambda): B(\lambda) f(\lambda) \in$ $D(A(\lambda))$ and $(A . B)(\lambda) f(\lambda)=A(\lambda) B(\lambda) f(\lambda)$, which implies $(A . B)(\lambda)=$ $A(\lambda) . B(\lambda)$ 。

\section{d) Hilbertian adjoint-maximal operators}

In the paragraph 2.4 we saw that not only minimal operators can be decomposed into a direct integral but also their hilbertian adjoints which are maximal operators.

However, if we decompose some $A^{*}(A \in \mathfrak{U})$, we are not able to prove that $A^{*}(\lambda)$ is $\mathscr{D}(\lambda)$-maximal but only that $A^{*}(\lambda) \subset A(\lambda)^{*}$ (this last one being maximal).

Consider indeed $(f, M A g)=(f, A M g)=\left(A^{*} f, M g\right), \forall f \in \mathscr{H}_{A^{*}}, \forall g \in$ $\mathscr{H}_{A}, \forall M \in \mathfrak{M}$ 。 By the integral decomposition we get for almost every $\lambda$ :

$$
|(f(\lambda), A(\lambda) g(\lambda))|=\left|\left(A^{*}(\lambda) f(\lambda), g(\lambda)\right)\right| \leq K \| g(\lambda)|| .
$$

This is true $\forall g \in \mathscr{H}_{A}$ and taking a dense set $\left\{g_{i}\right\}$ in $\mathscr{H}_{A}$ such that $\left\{g_{i}(\lambda)\right\}$ is dense in $\mathscr{H}_{A}(\lambda)$ and similarly a dense set $\left\{f_{i}\right\}$ in $\mathscr{H}_{A^{\prime}}$ such that $\left\{f_{i}(\lambda)\right\}$ is dense in $\mathscr{H}_{A^{+}}(\lambda)$, we get that for any $f(\lambda) \in \mathscr{H}_{A^{*}}(\lambda)$ : $A^{*}(\lambda) f(\lambda)=A(\lambda)^{*} f(\lambda)$. That means that, $\mathscr{H}_{A^{*}}(\lambda) \subset D\left(A(\lambda)^{*}\right)$ and that $A^{*}(\lambda)$ and $A(\lambda)^{*}$ coincide on $\mathscr{H}_{A^{*}}(\lambda)$ i. e. $A^{*}(\lambda) \subset A(\lambda) *$.

Applying this result to $A^{*}$ instead of $A$, we get $A^{\neq *}(\lambda) \subset\left(A^{\neq}(\lambda)\right)^{*}$ $=A(\lambda) * *$ 。

\section{e) The product}

Let $A, B \in \mathfrak{U}$ such that $A \square B$ is defined (it belongs to $\mathfrak{m}_{\sigma}^{\prime}$ )。

We have:

$$
\begin{aligned}
& \text { Range }(B \uparrow \mathscr{D}) \subset D\left(A^{\neq \uparrow}\right)=\mathscr{H}_{A^{\neq^{* *}}} \\
& \text { Range }\left(A^{*} \uparrow \mathscr{D}\right) \subset D\left(B^{*}\right)=\mathscr{H}_{B^{*}}
\end{aligned}
$$

In particular, $B$ maps $\mathscr{D}$ continuously in $\mathscr{H}_{A^{{ }^{*}}}$ i. e. there exists a finite set of elements $C_{1}, C_{2}, \ldots G_{N}$ in $\mathfrak{A}$ such that:

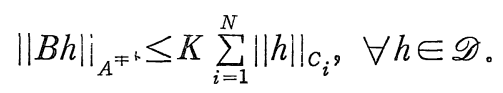

Notice that the set of norms $\left\{\|\cdot\|_{A^{*^{*}}}\right\}$ coincides on $\mathscr{D}$ with the set of norms $\left\{\|\bullet\|_{A} \mid A \in \mathfrak{U}\right\}$ and so, defines the same projective topology。 
Following exactly the same proof as in c) above, we get for almost every $\lambda \in \Lambda$ an inequality similar to the last one (with $B(\lambda), h(\lambda)$, $\left.A^{*^{*}}(\lambda), C_{i}(\lambda)\right)$ and so we conclude that the product $A(\lambda) \square B(\lambda)$ is well-defined. By integral decomposition we get easily that $(A \square B)(\lambda) \Upsilon_{\mathscr{D}_{0}(\lambda)}=A^{\neq^{*}}(\lambda) B(\lambda) \Upsilon_{\mathscr{D}_{0}(\lambda)}$ and with exactly the same proof as in c) (replacing $A(\lambda)$ by $A^{\neq *}(\lambda)$ and using he result of $\mathrm{d}$ )) we get finally: $(A \square B)(\lambda)=A(\lambda) \square B(\lambda)$ almost everywhere.

Remark. a) In the proof of this theorem, the existence of the set $\mathscr{D}_{0}$ dense in $\mathscr{D}$ for the projective topology and such that $\mathscr{D}_{0}(\lambda)$ is dense in $\mathscr{D}(\lambda)$, has been used in b), c) and e) i. e. for the sum, the products . and $\square$. For the part concerning the adjoints $*$ and $\neq$, we only need the existence of a dense set $\left\{g_{i}\right\}$ in $\mathscr{H}_{A}\left(\right.$ resp. $\left.\mathscr{H}_{A^{*}}, \mathscr{H}_{A \neq}\right)$ such that $\left\{g_{i}(\lambda)\right\}$ is dense in $\mathscr{H}_{A}(\lambda)$ (resp. $\mathscr{H}_{A^{*}}(\lambda), \mathscr{H}_{A^{\ddagger}}(\lambda)$ ). In particular, this set need not be the same for $A$ and $A^{\neq}$or $A$ and $A^{*}$.

4. 2. In conclusion of this section, we summarize the situation:

Theorem. Let $\mathfrak{X}$ be a countable $\neq-$ invariant set (resp. a partial $*_{-a l g e b r a}$ or an $O p^{*}$-algebra) of $\mathscr{D}$-minimal closed operators in a separable Hilbert space $\mathscr{H}$. There exists a Hilbert space $\mathscr{\mathscr { H }}$ containing $\mathscr{H}$ as closed subspace and a direct integral decomposition $\widehat{\mathscr{H}}=\int_{\Lambda} \mathscr{H}(\lambda) d \mu(\lambda)$ where $\mu$ is a regular Borel measure on a compact space $\Lambda$.

For almost every $\lambda \in \Lambda$, there exists a dense domain $\mathscr{D}(\lambda)$ in $\mathscr{H}(\lambda)$ and a countable $\neq-$ invariant set $\mathfrak{U}(\lambda)$ (resp. a partial $*_{\text {-algebra or an }}$ $O p^{*}$-algebra) of $\mathscr{D}(\lambda)$-minimal closed operators such that

$$
\forall f \in \mathscr{D}, \forall A \in \mathfrak{X} \quad A f=\int_{\Lambda} A(\lambda) f(\lambda) d \mu(\lambda)
$$

where $A(\lambda) \in \mathfrak{X}(\lambda)$ and $f(\lambda) \in \mathscr{D}(\lambda)$.

The decomposition is irreducible i.e. $(\mathfrak{A}(\lambda))_{w}^{\prime}$ is trivial for almost every $\lambda \in \Lambda$.

Remark. The proof of the irreducibility is the same as in [15] and follows from the choice of $\mathrm{m}$ as indicated above. 


\section{Part two: Decomposition of Uncountable Sets, Representations and States}

\section{$\S \mathbb{V}$. Separable $\neq-$ Invariant Sets}

5. 1. In this section, we are going to extend the result of integral decomposability from countable to uncountable (but separable) $\neq$-invariant sets.

In the previous sections, the fact that the graph-topology on $\mathscr{D}$ was metrizable, was very important because it allowed us to define the domain $\mathscr{D}(\lambda)$ and to show that it was dense in $\mathscr{H}(\lambda)$ for almost every $\lambda \in \Lambda$.

If we had considered an uncountable set of norms on $\mathscr{D}$, we would have been able to define $\mathscr{D}(\lambda)$ but not to prove that it was different from zero.

For this reason, although we shall consider now uncountable sets $\mathfrak{A}$, we shall assume that $\mathfrak{A}$ is dominated by a countable subset $\mathfrak{A}_{0}[6]$ i. e. for every $A \in \mathfrak{Q}$ there exists $B \in \mathfrak{A}_{0}$ such that $\|A f\| \leq K\|B f\|, \forall f \in \mathscr{D}$.

It follows from this assumption that the projective topology $t_{\mathfrak{x}}$ on $\mathscr{D}$ is equivalent to the projective topology $t_{\mathfrak{r}_{0}}$ (which is metrizable) and we assume $\mathscr{D}$ to be complete with respect to those topologies (i. e. $\mathfrak{U}$ and $\mathfrak{A}_{0}$ are both closed $\neq$-invariant sets).

\section{2. Topology on $\mathfrak{A}$}

It is possible to consider various topologies on a $\neq$-invariant set. Here we shall consider the so-called strong ${ }^{*}$-topology (shortly $s^{*}$ topology) [24] which is defined by the following set of semi-norms:

$$
A \in \mathfrak{X} \sim q_{f}(A)=\max \quad\left\{\|A f\|,\left\|A^{+} f\right\|\right\},
$$

$f$ running over $\mathscr{D}$. This topology is a particular case of quasi-uniform topologies which were introduced for Op*-algebras [4] [22] and it possesses good properties with respect to commutants and bicommutants [24] [27].

We shall assume that $\mathfrak{A}$ is separable in the $s^{*}$-topology i.e. there exists a countable set $\mathfrak{B}_{0}$ dense in $\mathfrak{A}$ for this topology.

Notice that we have made two assumptions on $\mathscr{D}$ which give us two countable subsets: $\mathfrak{A}_{0}$ which is a dominating subset and $\mathfrak{B}_{0}$ which 
is dense in $\mathfrak{A}$ for the $s^{*}$-topology。 We may assume $\mathfrak{N}_{0}=\mathfrak{B}_{0}$ otherwise we consider $\mathfrak{A}_{0} \cup \mathfrak{B}_{0}$ which is at the same time dense and dominating.

The $s^{*}$-topology is very natural to consider because if $\mathfrak{A}_{0}$ is dense in $\mathfrak{A}$ for this topology, $\mathfrak{A}$ and $\mathfrak{A}_{0}$ will have the same unbounded and bounded commutants:

$$
\left(\mathfrak{N}_{0}\right)_{\sigma}^{\prime}=\mathfrak{U}_{\sigma}^{\prime}[24]\left(\mathfrak{H}_{0}\right)_{w}^{\prime}=\mathfrak{H}_{w}^{\prime} \text { and }\left(\mathfrak{H}_{0}\right)_{s}^{\prime}=\mathfrak{X}_{s}^{\prime}
$$

(similar proof to [18] Lemma 2.2.2).

In particular, if we choose $\mathfrak{m} \subset \mathfrak{A}_{s}^{\prime}$ such that $\mathfrak{m}=\mathfrak{X}_{w}^{\prime} \cap \mathfrak{m}^{\prime}$, the same relations will hold for $\mathfrak{A}_{0}$ instead of $\mathfrak{A}$ so that we may apply the decomposition method of part I to $\mathfrak{A}_{0}$.

Moreover, the irreducibility of the $\mathfrak{A}(\lambda)$ we shall obtain later, will be implied by the irreducibility of the $\mathfrak{U}_{0}(\lambda)$.

We may also consider on $\mathscr{A}$ a weaker topology defined by the same semi-norms as the $s^{*}$-topology but when $f$ is restricted to be in $\mathscr{D}_{0}$ (the countable set dense in $\mathscr{D}$ for the projective topology that we introduced in 3.2).

Since $\mathscr{D}_{0}$ is countable, this topology (call it $\tau$ ) will be metrizable and $\mathfrak{A}$ will also be separable for it.

\section{3. Topology on $\mathfrak{2}_{0}(\lambda)$}

Let again $\mathfrak{A}$ be a $\neq$-invariant set with a $s^{*}$-dense, countable dominating subset $\mathfrak{H}_{0}$. Since $\mathfrak{U}_{0}$ is countable, we may decompose it by the method of part I and we get irreducible $\neq$-invariant sets $\mathfrak{A}_{0}(\lambda)$ on dense domains $\mathscr{D}(\lambda)$ of $\mathscr{H}(\lambda)$ with $\mathscr{D}(\lambda)$ complete for the $\mathfrak{N}_{0}(\lambda)$-graph-topology。

Consider on $\mathfrak{A}_{0}(\lambda)$ a topology $\tau_{\lambda}$ (analogous to $\tau$ above) defined by the following semi-norms:

$$
q_{\lambda, e_{i}(\lambda)}(A(\lambda))=\max \left\{\left\|A(\lambda) e_{i}(\lambda)\right\|, \| A^{ \pm}(\lambda) e_{i}(\lambda) i \mid\right\},
$$

where $e_{i} \in \mathscr{D}_{0}$. Since $\mathscr{D}_{0}$ is countable, this topology is metrizable.

Let us try to determine the completion of $\mathfrak{A}_{0}(\lambda)$ with respect to this topology.

For this, consider $\left\{A^{k}(\lambda)\right\}$ a Cauchy sequence in $\mathfrak{A}_{0}(\lambda)$ for the topology $\tau_{\lambda}$. We have $\left.\| A^{k}(\lambda)-A^{j}(\lambda)\right) e_{i}(\lambda) \|<\varepsilon$ and $\|\left(A^{k \neq}(\lambda)-\right.$ $\left.A^{-j \neq}(\lambda)\right) e_{i}(\lambda) \|<\varepsilon$ for all $e_{i} \in \mathscr{D}_{0}$. Hence the limits $h_{i}(\lambda)=\lim _{k} A^{k}(\lambda) e_{i}(\lambda)$ and $g_{i}(\lambda)=\lim _{k} A^{k \neq}(\lambda) e_{i}(\lambda)$ exist for $i=1,2, \ldots$ and those two relations define two operators $T(\lambda)$ and $S(\lambda)$ on $\mathscr{D}_{0}(\lambda)$ such that: 


$$
T(\lambda) e_{i}(\lambda)=h_{i}(\lambda), S(\lambda) e_{i}(\lambda)=g_{i}(\lambda), i=1,2, \ldots
$$

and moreover $S(\lambda)=T(\lambda) * \uparrow_{\mathscr{D}_{0}(\lambda)}$. In general, such operators need not be continuous on $\mathscr{D}_{0}(\lambda)$ for the $\mathscr{N}_{0}(\lambda)$-graph-topology. However, those which are actually continuous may be extended to $\mathscr{D}(\lambda)$ and we get $S(\lambda)=T(\lambda) *\lceil\mathscr{D}(\lambda)$. In that case taking the closure of those operators, we get $\mathscr{D}(\lambda)$-minimal operators $T(\lambda)$ and $S(\lambda)$ such that $S(\lambda)=T(\lambda)^{\ddagger}$. By construction, such operators may be approximated by elements of $\mathfrak{U}_{0}(\lambda)$ on $\mathscr{D}_{0}(\lambda)$. At this point, this whole construction may seem a bit complicated but, as we shall see in a moment, when we decompose any element $A \in \mathfrak{Y} \backslash \mathfrak{Y}_{0}$ we get exactly the same type of operators $A(\lambda)$ as the $T(\lambda)$ we just described.

5.4. Theorem. Let It be a $\neq$-invariant set with a countable doninating subset $\mathfrak{I}_{0}$ dense in $\mathfrak{U}$ for the $s^{*}$-topology. Let the countable set $\mathfrak{U}_{0}$ be decomposed in

$$
\mathfrak{U}_{0}=\int_{\Lambda} \mathfrak{Y}_{0}(\lambda) d \mu(\lambda)
$$

where $\mathscr{V}_{0}(\lambda)$ is a countable set of $\mathscr{D}(\lambda)$-minimal operators in $\mathscr{H}(\lambda)$ (following the method of part I).

On the other hand, let $A \in \mathfrak{A} \backslash \mathfrak{\mathfrak { Y } _ { 0 }}$ be decomposed as an individual closed operator in $A=\int_{\Lambda} A(\lambda) d \mu(\lambda)$ where $A(\lambda)$ is a closed operator in $\mathscr{H}(\lambda)$. Then, for almost every $\lambda \in \Lambda, A(\lambda)$ is a $\mathscr{D}(\lambda)$-minimal operator and $A(\lambda) \in \overline{\mathfrak{Y}}_{0}(\lambda)^{\tau} \lambda$.

Proof。 a) Because $\left(\mathfrak{U}_{0}\right)_{\sigma}^{\prime}=\mathfrak{U}_{\sigma}^{\prime}$, we have

$$
\mathfrak{U}_{0} \subset \mathfrak{U} \subset\left(\mathfrak{U}_{0}\right)_{\sigma \sigma}^{\prime \prime}=\mathfrak{U}_{\sigma \sigma}^{\prime \prime} \subset \mathfrak{M}_{\sigma}^{\prime}
$$

Hence, any element of $\mathfrak{X}$ is decomposable (2.4). Since the topologies $t_{\mathfrak{A}}$ and $t_{\mathfrak{श}_{0}}$ are equivalent on $\mathscr{D}, A$ is continuous from $\mathscr{D}\left[t_{\mathfrak{r}_{0}}\right]$ into $\mathscr{H}$ so the operators $A(\lambda)$ occurring in the integral decomposition are continuous from $\mathscr{D}(\lambda)\left[\iota_{\mathfrak{\Re}_{0}(\lambda)}\right]$ into $\mathscr{H}(\lambda)$. Moreover, $\mathscr{D}(\lambda)$ and $\mathscr{D}_{0}(\lambda)$ are cores for $A(\lambda)$ almost everywhere (see last theorem of 3.3).

b) Since $\mathfrak{A}$ is separable in the $s^{*}$-topology and a fortiori in the topology $\tau$, there exists a Cauchy sequence $\left\{A^{i}\right\}$ in $\mathscr{V}_{0}$ such that $q_{e_{j}}\left(A^{i}-A\right) \rightarrow 0$ for $e_{j} \in \mathscr{D}_{0}, j=1,2, \ldots$ This means in particular that $\forall e_{j} \in \mathscr{D}_{0}$ : 


$$
\left\|\left(A^{i}-A\right) e_{j}\right\| \rightarrow 0 \text { and }\left\|\left(A^{i \neq}-A^{\neq}\right) e_{j}\right\| \rightarrow 0 .
$$

Decomposing those norms in direct integrals, we get that there exists a subsequence $\left\{A^{i}\right\} \subset \mathfrak{U}_{0}$ such that for almost every $\lambda \in \Lambda$ :

$$
\begin{aligned}
& \left\|\left(A^{i_{k}}(\lambda)-A(\lambda)\right) e_{j}(\lambda)\right\| \rightarrow 0 \text { and } \\
& \left\|\left(A^{i_{k^{*}}}(\lambda)-A^{\neq}(\lambda)\right) e_{j}(\lambda)\right\| \rightarrow 0, \forall e_{j}(\lambda) \in \mathscr{D}_{0}(\lambda) .
\end{aligned}
$$

Since there is only a countable number of such norms (indexed by j) we may choose the subsequence $\left\{A^{i} k\right.$ independent of $j$ and suitable for the adjoints as well. Finally, we have that $q_{\lambda, e_{j}(\lambda)}\left(A^{i_{k}}(\lambda)\right.$ $-A(\lambda)) \rightarrow 0$ for $j=1,2, \ldots$ i. e. $A(\lambda)$ belongs to the completion of $\mathfrak{U}_{0}(\lambda)$ with respect to the topology $\tau_{\lambda}$.

In conclusion, all elements of $\mathfrak{U}$ are decomposed into $\mathscr{D}(\lambda)$-minimal operators which may be approximated on $\mathscr{D}_{0}(\lambda)$ by elements of $\mathfrak{U}_{0}(\lambda)$. Moreover, they are continuous from $\mathscr{D}(\lambda)$ into $\mathscr{H}(\lambda)$.

5.5. Conclusion. Define in $\overline{\mathfrak{Y}}_{0}(\lambda)^{\tau} \cap \mathfrak{S}(\mathscr{D}(\lambda))$ the subset $\mathfrak{U}(\lambda) \equiv$ $\{A(\lambda) \mid A \in \mathfrak{Q}\}$ consisting in the images of the elements of $\mathfrak{U}$ after decomposition. Then $\mathfrak{A}(\lambda)$ is a $\neq$-invariant set of $\mathscr{D}(\lambda)$-minimal operators. We have thus decomposed the $\neq-$ invariant set $\mathfrak{A}$ into irreducible $\neq-$-invariant sets $\mathfrak{U}(\lambda)$. Moreover, $\mathfrak{U}(\lambda)$ is "generated" by $\mathfrak{A}_{0}(\lambda)$ in the same way as $\mathfrak{A}$ was "generated" by $\mathfrak{U}_{0}$.

Since the algebraic relations pass through the integral decompositions for almost every $\lambda \in \Lambda$, if we begin with a partial $*_{\text {-algebra, we }}$

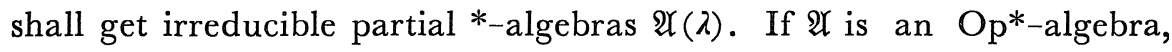
the $\mathfrak{X}(\lambda)$ 's will be Op*-algebras as well $(A(\lambda) \in \mathfrak{A}(\lambda)$ will be continuous from $\mathscr{D}(\lambda)$ into itself).

This last result about decomposition of Op*-algebras generalizes the result we got in [18] where we made the stronger assumption that $\mathfrak{A}$ was separable for some quasi-uniform topology [22] which is actually a finer topology than the $s^{*}$-topology we consider here.

\subsection{Particular cases. $\mathbb{V}^{*}$-sets and $V^{*}$-algebras}

In an attempt to generalize the von Neumann theory to unbounded operators, special attention has been paid in [25] to a class of Op*algebras satisfying $\mathfrak{A}=\mathfrak{U}_{\sigma \sigma}^{\prime \prime}$ (called $V^{*}$-algebras) or satisfying $\mathfrak{A}=\mathfrak{A}_{w \sigma}^{\prime \prime}$ 
(called $S V^{*}$-algebras). Several interesting results have been obtained for those classes of algebras. Here, since we are not restricted to Op*-algebras, we shall call a $V^{*}$-set (resp. a $S V^{*}$-set), a $\neq$-invariant subset $\mathfrak{A}$ of $\mathfrak{E}$ such that $\mathfrak{X}=\mathfrak{U}_{\sigma \sigma}^{\prime \prime}\left(\right.$ resp. $\left.\mathfrak{U}=\mathfrak{X}_{w \sigma}^{\prime \prime}\right)$. In particular, such sets are closed for the $s^{*}$-topology [24].

If we assume that a $V^{*}$-set possesses a countable dominating subset $\mathfrak{N}_{0}$, dense for the $s^{*}$-topology, we have in fact: $\mathfrak{A}=\overline{\mathfrak{U}}_{0}^{s^{*}}=\overline{\mathfrak{A}}^{s^{*}}=\left(\mathfrak{A}_{0}\right)_{\sigma \sigma}^{\prime \prime}=\mathfrak{A}_{\sigma \sigma}^{\prime \prime}$.

If we decompose $\mathfrak{A}$ in a direct integral of $\neq$-invariant sets $\mathfrak{A}(\lambda)$ as above, it is more coherent to consider instead of the $\mathfrak{A}(\lambda)$ their bicommutants $\mathfrak{X}(\lambda)_{\text {\%o }}^{\prime \prime}$ which are (a fortiori irreducible) $V^{*}$-sets in (E) $(\mathscr{D}(\lambda))$. For every $A \in \mathfrak{A}$, we shall have $A(\lambda) \in \mathfrak{A}(\lambda)_{\sigma_{0}}^{\prime \prime}$

Similarly, if we decompose a $S V^{*}$-set in a direct integral, we shall consider $\mathfrak{A}(\lambda)_{\text {wo }}^{\prime \prime}$ which is equal to $\mathfrak{C}(\mathscr{D}(\lambda))$ i。 e. an irreducible $S V^{*}$-set.

Thus, $V^{*}$-sets (resp. $S V^{*}$-sets) may be decomposed into irreducible $V^{*}$-sets (resp. $S V^{*}$-sets).

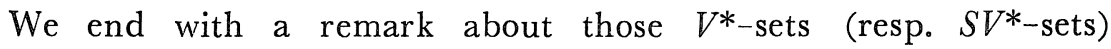
possessing a countable subset $\mathfrak{A}_{0}$ dense for the $s^{*}$-topology。

As we said above, if $\mathfrak{A}_{0}$ is dense in $\mathfrak{A}=\mathfrak{U}_{\sigma \sigma}^{\prime \prime}$, we get $\left(\mathfrak{H}_{0}\right)_{\sigma \sigma}^{\prime \prime}=\overline{\mathfrak{Y}}_{0}^{s *}$. Conversely, if $\left(\mathfrak{A}_{0}\right)_{\sigma o}^{\prime \prime}=\overline{\mathfrak{A}}_{0}^{s^{s}}$, then $\mathfrak{A} \equiv\left(\mathfrak{H}_{0}\right)_{\text {oo }}^{\prime \prime}$ is a $V^{*}$-set separable in the $s^{*}$-topology. Hence we get:

Proposition. a) The $V^{*}$-sets separable in the $s^{*}$-topology are exactly the unbounded bicommutants of countable sets $\mathfrak{H}_{0}$ satisfying $\overline{\mathfrak{A}}_{0}^{s^{*}}=\left(\mathfrak{H}_{0}\right)_{o \text { o. }}^{\prime \prime}$

(Notice that this last condition is not at all automatic for unbounded operators [30]. It holds if, for instance, $\mathfrak{A}_{0}$ consists in bounded operators only).

b) Similarly, the $S V^{*}$-sets separable in the $\boldsymbol{s}^{*}$-topology are exactly the bicommutants $\left(\mathfrak{Q}_{0}\right)_{\text {wo }}^{\prime \prime}$ of countable sets $\mathfrak{\mathscr { N }}_{0}$ satisfying $\overline{\mathfrak{R}}_{0}^{s^{*}}=\left(\mathfrak{H}_{0}\right)_{w_{0 \circ}}^{\prime \prime}$

We mention this characterization of separable $V^{*}$-sets and $S V^{*_{-}}$ sets because it reminds us about the characterization of von Neumann algebras in a separable Hilbert space as the bicommutant of a countable number of projections. 


\section{$\S$ VI. Representations of $\mathbb{P a r t i a l} *$-Algebras}

6.1. The decomposition method we have discussed all along this paper will be used in this section to decompose representations of abstract partial *-algebras into direct integral of irreducible representations. We first recall some definitions following [1], [2], [3] and [12].

Definition. A partial *-algebra is a complex vector space with an involution $A \rightarrow A^{+}$and a subset $\Gamma \subset \mathfrak{U} \times \mathfrak{A}$ such that:

1) $(A, B) \in \Gamma$ implies $\left(B^{+}, A^{+}\right) \in \Gamma$

2) $\left(A, B_{1}\right) \in \Gamma$ and $\left(A, B_{2}\right) \in \Gamma$ implies $\left(A, B_{1}+\lambda B_{2}\right) \in \Gamma, \forall \lambda \in \mathbb{C}$

3) If $(A, B) \in \Gamma$, there exists an element $A o B \in \mathfrak{X}$ such that:

$$
A o(B+C)=(A o B)+(A o C) \text { and }(A o B)^{+}=B^{+} o A^{+} .
$$

Remark. this partial product $o$ need not be associative.

In the sequel, we shall assume that the partial $*_{\text {-algebra }} \mathscr{A}$ is provided with a locally convex topology for which the involution + is continuous.

We collect some definitions about representations of partial *-algebras :

6.2. Definitions [3]. A representation $\pi$ of $\mathfrak{A}$ is a homomorphism from $\mathfrak{A}$ into the minimal closed operators $\mathfrak{S}(\mathscr{D})$ of a dense domain $\mathscr{D}$ of a separable Hilbert space $\mathscr{H}$, i. e. $\pi(A+\lambda B)=\pi(A) \uparrow \lambda \pi(B)$, $\pi\left(A^{+}\right)=(\pi(A))^{\neq}$and if $(A, B) \in \Gamma$, either $\pi(A o B)=\pi(A) . \pi(B)$ or $\pi(A o B)=\pi(A) \square \pi(B)$ 。

In the first case, $\pi$ is called a hermitian representation (i. e. a representation of $\mathfrak{A}$ in $\mathfrak{C}^{s}(\mathscr{D})$ in the notations of section $\left.I\right)$, in the second case, $\pi$ is called a weakly hermitian representation (i. e. in (ㄴw $(\mathscr{D}))$.

Definitions. As usual, a representation $\pi$ of $\mathfrak{A}$ is said to be closed if $\mathscr{D}=\overline{\mathscr{D}}$ i. e. $\mathscr{D}$ is complete with respect to the projective topology defined by all the graph-norms $\|\cdot\|_{\pi(A)}, \forall A \in \mathscr{A}$ (in this 
section, we shall call $t_{\pi}$ this topology). Moreover, since we are dealing with more general sets than Op*-algebras, we must also consider another notion of "closure" which has been introduced in [2]: A representation $\pi$ is fully closed if

$$
\mathscr{D}=\mathscr{D}(\pi(\mathfrak{U}))=\underset{A \in \mathscr{R}}{\bigcap} D(\pi(A)) .
$$

It has been proved in [3] that any hermitian representation of a partial *-algebra admits a unique minimal closed hermitian extension on $\overline{\mathscr{D}}$ and a unique minimal fully closed, but weakly hermitian extension to $\mathscr{D}(\pi(\mathfrak{R}))$. In particular, we may always assume $\pi$ to be closed but not necessarily fully closed.

Definitions. As usual, a vector $\Omega \in \mathscr{D}$ is called cyclic for $\pi$ if the set $\pi(\mathfrak{U}) \Omega$ is dense in $\mathscr{H}$. Moreover, a vector $\Omega \in \mathscr{D}$ is called strongly cyclic for $\pi[3]$ if the set $\{\pi(X) \Omega \mid A o X$ is defined $\forall A \in \mathfrak{N}\}$ is firstly contained in $\bar{D}$ and secondly is dense in it for $t_{\pi}$. (In that situation, $X$ is called a universal right-multiplier in $\mathfrak{U})$. This notion of strongly cyclic vector gets back to the one of Powers [6] for Op*-algebras because, in that case, any $A \in \mathfrak{A}$ is a universal rightmultiplier.

Definition. The representation $\pi$ is called strongly continuous if the map $A \rightarrow\|\pi(A) f\|$ is continuous from $\mathfrak{A}$ into $\mathscr{H}$, for every $f \in \mathscr{D}$. Notice that, because the involution is continuous in $\mathfrak{A}$, a strongly continuous representation is automatically strongly $*_{\text {-continuous. }}$

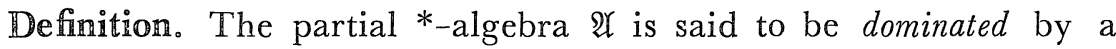
subset $\mathfrak{Y}_{0}$ if for any representation $\pi$ of $\mathfrak{A}$, the set $\pi\left(\mathfrak{Y}_{0}\right)$ is a dominating subset of $\pi(\mathfrak{U})$ in the sense we used in 5.1.

In the sequel, we shall also assume that $\mathfrak{A}$ is separable in its own topology i.e., there exists a countable subset $\mathfrak{U}_{0}$ dense in $\mathfrak{U}_{0}$

As before, we may find a subset $\mathfrak{U}_{0}$ which is at the same time dense and dominating.

6.3. Theorem. Let $\mathfrak{A}$ be a separable locally convex partial *algebra dominated by a countable subset $\mathfrak{N}_{0}$. Let $\pi$ be a strongly continuous 
representation of $\mathfrak{X}$ by minimal closed operators on a dense domain $\mathscr{D}$ of a separable Hilbert space $\mathscr{H}$. Then there exists a separable Hilbert space $\mathscr{\mathscr { H }}$ containing $\mathscr{H}$ as closed subspace and a direct integral decomposition

$$
\widehat{\mathscr{H}}=\int_{\Lambda} \mathscr{H}(\lambda) d \mu(\lambda)
$$

where $\mu$ is a positive Borel measure on a compact space 1 . There exists for almost every $\lambda \in \Lambda$ a representation $\pi_{\lambda}$ of $\mathfrak{A}$ by minimal operators on a dense domain $\mathscr{D}(\lambda)$ of $\mathscr{H}(\lambda)$ such that:

1) $\forall f \in \mathscr{D}, \forall A \in \mathfrak{R}, \pi(A) f=\int_{\Lambda} \pi_{\lambda}(A) f(\lambda) d \mu(\lambda)$;

2) $\left(\pi_{\lambda}(\mathfrak{R})\right)_{w}^{\prime}=\mathbb{C}$ i.e. $\pi_{\lambda}$ is irreducible;

3) If $\pi$ is hermitian, $\pi_{\lambda}$ is hermitian;

If $\pi$ is weakly hermitian, $\pi_{\lambda}$ is weakly hermitian;

4) Although the $\pi_{\lambda}$ are not necessarily strongly continuous representations, they have some kind of continuity property:

If $A=\lim _{\alpha} A^{\alpha}$ in $\mathfrak{A}$ there exists a sequence $\left\{A^{i}\right\} \subset\left\{A^{\alpha}\right\}$ such that $\left\|\left(\pi(A)-\pi\left(A^{i}\right)\right) f\right\| \rightarrow 0$ and a subsequence $\left\{A^{i_{k}}\right\}$ such that $\|\left(\pi_{\lambda}\left(A^{i_{k}}\right)-\right.$ $\left.\pi_{\lambda}(A)\right) f(\lambda) \| \rightarrow 0$ for every $f \in \mathscr{D}_{0}$ (the dense set in $\mathscr{D}$ for the projective topology);

5) If $\Omega \in \mathscr{D}$ is cyclic (resp. strongly cyclic) for $\pi$, then there exist $\Omega(\lambda) \in \mathscr{D}(\lambda)$ which are cyclic (resp. strongly cyclic) for $\pi_{\lambda}$.

Proof. The proof of 1) and 2) is exactly the same as what we did in [18] for Op*-algebras, excepted that we consider here the $s^{*}$-topology instead of a quasi-uniform one. This proof consists in showing that the maximal extension [15] $\hat{\pi}$ on $\hat{\mathscr{D}} \subset \mathscr{\mathscr { H }}$ possesses the same properties as $\pi$ on $\mathscr{D} \subset \mathscr{H}$, essentially that $\hat{\mathscr{D}}$ is metrizable (this is because $\mathfrak{A}_{0}$ is dominating) and that $\hat{\pi}(\mathfrak{U})$ is separable in the $s^{*}$-topology (see [18] for the details). Finally, we apply the decomposition of the previous sections to $\hat{\pi}(\mathfrak{R})$ which induces a decomposition of $\pi(\mathfrak{A})$.

The proof of 3) follows from the fact that if $X . Y$ is defined, then $(X . Y)(\lambda)=X(\lambda) . Y(\lambda)$ almost everywhere and similarly, if $X \square Y$ is defined, $(X \square Y)(\lambda)=X(\lambda) \square Y(\lambda)$ almost everywhere. Hence, defining $\pi_{\lambda}(A) \equiv(\pi(A))(\lambda)$, the map $\pi_{\lambda}: \mathscr{U} \rightarrow \mathbb{C}(\mathscr{D}(\lambda))$ is a homomorphism from $\mathfrak{A}$ into $\mathfrak{C}^{s}$ or $\mathfrak{J}^{w}$, hence, defines a hermitian or weakly hermitian 
representation of $\mathfrak{U}$.

Proof of 4). If $A^{\alpha} \rightarrow A$ in 2 , we have $\pi(A)=\lim _{\alpha} \pi\left(A^{\alpha}\right)$ in $\mathfrak{C}(\mathscr{D})$ for

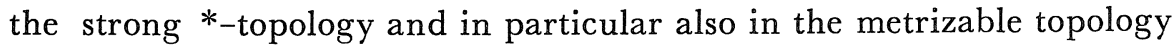
$\tau$ introduced in 5.2. We have:

$$
\begin{aligned}
& q_{e_{j}}\left(\pi\left(A^{\alpha}\right)-\pi(A)\right)=\max \left\{\left\|\left(\pi\left(A^{\alpha}\right)-\pi(A)\right) e_{j}\right\|_{,}\right. \\
& \left.\left\|\left(\pi\left(A^{\alpha}\right)^{\neq}-\pi(A)^{\neq}\right) e_{j}\right\|\right\}, \forall e_{j} \in \mathscr{D}_{0}, \quad j=1,2 \ldots
\end{aligned}
$$

But since this topology is metrizable, we may extract from the net $\left\{A^{\alpha}\right\}$ a sequence $\left\{A^{i}\right\}$ converging to $A$. We have thus $\left\|\left(\pi\left(A^{i}\right)-\pi(A)\right) e_{j}\right\|$ $\rightarrow 0$ when $i \rightarrow \infty$ and for every $e_{j} \in \mathscr{D}_{0}$. Decomposing this norm in a direct integral, we get that there exists a subsequence $\left\{A^{i_{k}}\right\}$ such that for almost every $\lambda \in \Lambda:\left\|\left(\pi_{\lambda}\left(A^{i}\right)-\pi_{\lambda}(A)\right) e_{j}(\lambda)\right\| \rightarrow 0$ (since there is only a countable number of such norms, indexed by $j$, the subsequence may be chosen independent of $j$ ). This proves point 4) and, in fact this means that $\left\{\pi_{\lambda}\left(A^{i_{k}}\right)\right\}$ tends to $\pi_{\lambda}(A)$ in the topology $\tau_{\lambda}$ considered in 5.3 .

Proof of 5). The fact that $\Omega \in \mathscr{D}$ is cyclic for $\pi$ means that $\pi(\mathscr{U}) \Omega$ is dense in $\mathscr{H}$. But, if $f \in \mathscr{H}$ is a limit of some sequence $\pi\left(A^{i}\right) \Omega,\left\{A^{i}\right\} \subseteq \mathfrak{X}$, then there exists a subsequence $\left\{A^{i}{ }^{k}\right\}$ such that for almost every $\lambda \in \Lambda: f(\lambda)=\lim _{i} \pi_{\lambda}\left(A^{i_{k}}\right) \Omega(\lambda)$. Thus, the set $\pi_{\lambda}(\mathfrak{X}) \Omega(\lambda)$ is dense in the set of $f(\lambda) \in \mathscr{H}(\lambda)$ which are the images of some $f \in \mathscr{H}$. Since we know that this last set is dense in $\mathscr{H}(\lambda)$, it follows that $\Omega(\lambda)$ is cyclic for $\pi_{\lambda}$.

Let now $\Omega \in \mathscr{D}$ be strongly cyclic for $\pi$. Every $g \in \mathscr{D}$ is a limit of the type $g=\lim _{i} \pi\left(X^{i}\right) \Omega$ such that $\pi(A) g=\lim _{i} \pi\left(A o X^{i}\right) \Omega$ for every $A \in \mathfrak{R}$, where the $X^{i}$ 's are universal right multipliers. That means $g$ is limit of $\pi\left(X^{i}\right)$ in the topology $t_{\pi} \equiv t_{\pi(\mathfrak{r})}$. But, by assumption, this topology is metrizable since it is equivalent to $t_{\pi\left(\mathfrak{n}_{0}\right)}$ where $\mathfrak{U}_{0}$ is the countable dominating subset of $\mathfrak{X}$. Because of this, we may extract a subsequence $\left\{X^{i}\right\}$ independent of $A$ such that $g(\lambda)=\lim \pi_{\lambda}\left(X^{i_{k}}\right) \Omega(\lambda)$ and $\pi_{\lambda}(A) g(\lambda)=\lim _{i_{k}} \pi_{\lambda}\left(A o X^{i_{k}}\right) \Omega(\lambda), \quad \forall A \in \mathfrak{U}$, i. e. $g(\lambda)$ is a limit of this type in the topology $t_{\pi\left(\mathfrak{\Re}_{0}\right)}$ which is equivalent to $t_{\pi(\mathfrak{R})}$.

Moreover, since such $g(\lambda)$ 's (coming from $g \in \mathscr{D}$ ) are dense in 
$\mathscr{D}(\lambda)$ for $t_{\pi}$ (because $\mathscr{D}_{0}(\lambda)$ is contained in this set of $g(\lambda)$ 's), every element of $\mathscr{D}(\lambda)$ is a limit of the type above. Hence $\Omega(\lambda)$ is strongly cyclic for $\pi_{\lambda}$.

\section{§ VII. Decomposition of States}

7.1. Because the product of two elements of a partial *-algebra is not always defined, the notion of state has to be modified in consequence. Indeed, a state $\omega$ on an Op*-algebra or a $C^{*}$-algebra gives rise by the GNS construction to a representation in a Hilbert space. The scalar product of this last one is given by

$$
\left\langle\phi_{A}, \phi_{B}\right\rangle=\omega\left(A^{+} \circ B\right), A, B \in \mathfrak{A}, \phi_{A}, \phi_{B} \in \mathscr{H}
$$

and thus, an explicit use of the product is made.

When we are working with partial *-algebras, the notion of state has to be replaced by the notion of $h$-state introduced in [3] (see also [29] for Op*-algebras) and which is in fact a sesquilinear form on $\mathfrak{A}$.

We recall the definitions we shall need in the sequel.

Definition. An $h$-form on a partial $*$-algebra $\mathfrak{A}$ is a sesquilinear form $\omega$ on $\mathfrak{A} \times \mathfrak{A}$ which is moreover positive $(\omega(A, A) \geq 0, \forall A \in \mathfrak{U})$ and multiplication invariant (if $A o C$ and $A^{+} o B$ are defined, then $\left.\omega\left(A^{+} o B, C\right)=\omega(B, A o C)\right)$. This $h$-form is called a $h$-state if moreover $\omega(e, e)=1$ (where $e$ is the unit element of $\mathfrak{A}$, and we shall assume it exists).

Beginning with a $h$-state $\omega$ on $\mathfrak{A}$, the authors of [3] [12] generalized the GNS construction provided two more conditions hold:

1) $\mathfrak{A}$ has to be semi-associative i. e. $\forall A, B, C \in \mathfrak{A}$ such that $C$ is a universal right-multiplier, we have: if $A o B$ is defined, then $A o(B o C)$ is defined and is equal to $(A o B) o C$.

2) $\omega$ is weakly $G N S$, which means that when we construct the Hilbert space $\mathscr{H}_{\omega}$ similarly as in the usual case, i.e. the completion of $\mathscr{U} / \operatorname{ker} \omega$ with respect to the scalar product $\left\langle\phi_{A}, \phi_{B}\right\rangle \equiv \omega(A, B)$, the set $\mathscr{R}_{\omega}=\left\{\phi_{X}: X\right.$ is a universal right-multiplier $\}$ is dense in $\mathscr{H}_{\omega}$.

Under those two assumptions, it has been proved [3] that $\omega$ gives 
rise in $\mathscr{H}_{\omega}$ to a fully closed, weakly hermitian, cyclic representation $\pi_{\omega}$ on $\mathscr{D}_{\omega}=\bigcap_{A \in \mathscr{U}} D\left(\pi_{\omega}(A)\right)$ such that $\omega(A, B)=\left\langle\pi_{\omega}(A) \Omega, \pi_{\omega}(B) \Omega\right\rangle$ (where $\Omega$ is the cyclic vector).

Moreover, the restriction of $\pi_{\omega}$ to $\mathscr{D} \equiv \overline{\mathscr{R}}_{\omega}$ (the completion of $\mathscr{R}_{\omega}$ with respect to $t_{\pi_{\omega}}$ ) is a strongly cyclic, closed, weakly hermitian representation of $\mathfrak{A}$ (We refer to [3] for all the details of the construction and of the proof).

Now we are in position to write the theorem about decomposition of $h$-states. In this theorem, we shall call an $h$-state extremal if it gives rise to a GNS representation $\pi_{\omega}$ whose weak bounded commutant is trivial. Moreover, since we are going to apply to the GNS representation the theorem 6.3 of previous section, it is necessary that this representation be strongly continuous, and this is obtained by considering jointly continuous $h$-states.

\subsection{Theorem}

Let $\mathfrak{O}$ be a semi-associative partial *-algebra dominated by a countable subset and separable in its own locally convex topology. Let $\omega$ be a jointly continuous, weakly GNS h-state on $\mathfrak{A}$. Then, there exists a regular Borel measure $\mu$ on a compact space $\Lambda$ and for almost every $\lambda \in \Lambda$, weakly GNS, h-states $\omega_{\lambda}$ on $\mathfrak{U}$ such that:

a) $\omega=\int_{\Lambda} \omega_{\lambda} d \mu(\lambda)$;

b) $\omega$ is extremal;

c) $\omega$ need not be jointly continuous, but if $A^{\alpha} \rightarrow A$ in $\mathfrak{N}$, there exists a sequence $\left\{A^{i}\right\} \subset\left\{A^{\alpha}\right\}$ such that $\omega\left(A^{i}, A^{i}\right) \rightarrow \omega(A, A)$ and there exists a subsequence $\left\{A^{i_{k}}\right\}$ such that $\omega_{\lambda}\left(A^{i_{k}}, A^{i_{k}}\right) \rightarrow \omega_{\lambda}(A, A)$, for almost every $\lambda \in \Lambda$;

d) $\operatorname{ker} \omega \subset \operatorname{ker} \omega_{\lambda}$ and ker $\pi_{\omega} \subset \operatorname{ker} \pi_{\omega, \lambda}$ 。

Proof. a) Consider the strongly-cyclic, closed, weakly hermitian GNS-representation $\pi_{\omega}$ on $\mathscr{D}=\overline{\mathscr{R}_{\omega}}$ described above and apply the integral decomposition of Theorem 6.3 to $\pi_{\omega}$ i. e. there exist domains $\mathscr{D}(\lambda)$ in $\mathscr{H}_{\omega}(\lambda)$ and weakly hermitian representations $\pi_{\omega, \lambda}$ of $\mathfrak{U}$ such that:

$$
\forall f \in \mathscr{D} \text { and } \forall G \in \mathfrak{Y}: \pi_{\omega}(G) f=\int_{\Lambda} \pi_{\omega, \lambda}(G) f(\lambda) d \mu(\lambda)
$$


In particular, $\Omega \in \mathscr{D}$ hence $\Omega(\lambda) \in \mathscr{D}(\lambda)$ for almost every $\lambda \in \Lambda$ and we have $\forall A, B \in \mathfrak{A}$ :

$$
\begin{aligned}
& \omega(A, B)=\left\langle\pi_{\omega}(A) \Omega, \pi_{\omega}(B) \Omega\right\rangle= \\
& \int_{\Lambda}\left(\pi_{\omega, \lambda}(A) \Omega(\lambda), \pi_{\omega, \lambda}(B) \Omega(\lambda)\right) d \mu(\lambda) \equiv \int_{\Lambda} \omega_{\lambda}(A, B) d \mu(\lambda),
\end{aligned}
$$

where we define

$$
\omega_{\lambda}(A, B) \equiv\left(\pi_{\omega, \lambda}(A) \Omega(\lambda), \pi_{\omega, \lambda}(B) \Omega(\lambda)\right) .
$$

We must now show that, so defined, the $\omega_{\lambda}$ are weakly GNS $h$-states on 2 . For this, we need to check four properties:

i) the positivity: $\left(\omega_{\lambda}(A, A) \geq 0\right)$ is obvious.

ii) the multiplication invariance: Let $A, B, C \in \mathfrak{X}$ such that $A o C$ and $A^{+} O B$ are defined. Since $\omega$ itself is multiplication-invariant $\omega\left(A^{+} o B, C\right)=\omega(B, A o C)$ i. e. since $\pi_{\omega}$ is weakly hermitian:

$$
\left\langle\left(\pi_{\omega}(A)^{\ddagger} \square \pi_{\omega}(B)\right) \Omega, \pi_{\omega}(C) \Omega\right\rangle=\left\langle\pi_{\omega}(B) \Omega,\left(\pi_{\omega}(A) \square \pi_{\omega}(C)\right) \Omega\right\rangle .
$$

This equality passes through the integral decomposition since the product $\square$ is decomposed correctly, and we get in this way the multiplication invariance of $\omega_{\lambda}$.

iii) By adding a normalization if necessary, it is always possible to get $\omega_{\lambda}(e, e)=1$.

Those three properties make the $\omega_{\lambda}$ 's into $h$-states on $\mathfrak{A}$. We still must show that they are weakly GNS.

iv) As we did in the proof of 5) of Theorem 6.3, the fact that $\left\{\phi_{X} \equiv \pi_{\omega}(X) \Omega \mid X\right.$ is a universal right-multiplier $\}$ is dense in $\mathscr{H}_{\omega}$ implies that $\left\{\pi_{\omega, \lambda}(X) \Omega(\lambda)\right\}$ is dense in $\mathscr{H}_{\omega}(\lambda)$ for almost every $\lambda \in \Lambda$. On the other hand, it is obvious from the way we have defined $\omega_{\lambda}$ that the GNS representation, $\pi_{\omega_{\lambda}}$ associated to it, is unitary equivalent to $\pi_{\omega, \lambda}$.

Remark. Remember that in all this, the domain $\mathscr{D}$, which is the completion of $\mathscr{R}_{\omega}$ with respect to $t_{\pi_{\omega}^{(\mathfrak{R})}}$ or equivalently to $t_{\pi_{\omega} \in\left(\mathfrak{R}_{0}\right)}$, is metrizable.

The domain $\mathscr{D}_{0}$, dense in $\mathscr{D}$, consists then in a countable set of elements of the form $e_{j}=\pi_{\omega}\left(X^{j}\right) \Omega$ where $X^{j}$ is a universal rightmultiplier of $\mathfrak{U}$.

b) trivial by Theorem 6.3.2).

c) follows from theorem 6.3.4) and the fact that we may assume 
that $\Omega \in \mathscr{D}_{0}$.

d) Let $A \in \operatorname{ker} \omega$. By integral decomposition, we get:

$$
0=\omega(A, A)=\int_{\Lambda} \omega_{\lambda}(A, A) d \mu(\lambda),
$$

which implies $\omega_{\lambda}(A, A)=0$ for almost every $\lambda \in \Lambda$ i. e. $A \in \operatorname{ker} \omega_{\lambda}$, $\forall \lambda \in \Lambda \backslash \mathscr{N}_{A}$, where $\mathscr{N}_{A}$ is a null-set depending on $A$. If we consider first such $A$ in $\mathfrak{N}_{0}$, we may get a common null-set $\underset{A \in \mathscr{I}_{0}}{\cup} \mathscr{N}_{A}$ i. e. $A \in \operatorname{ker} \omega \cap \mathfrak{X}_{0}$ implies that $A \in \operatorname{ker} \omega_{\lambda} \cap \mathfrak{A}_{0}$ for almost every $\lambda \in \Lambda$. Take now $A \in$ ker $\omega \cap\left(\mathfrak{U} \backslash \mathfrak{U}_{0}\right)$. There exists a net $\left\{A^{\alpha}\right\}$ in $\mathfrak{A}_{0}$ converging to $A$ and we know by the point c) above that there exist $\left\{A^{i}\right\}$ and $\left\{A^{i_{k}}\right\}$ such that $\omega\left(A^{i}, A^{i}\right) \rightarrow \omega(A, A)=0$ and $\omega_{\lambda}\left(A^{i_{k}}, A^{i_{k}}\right) \rightarrow \omega_{\lambda}(A, A)$ for almost every $\lambda \in \Lambda$. This implies that $\omega_{\lambda}(A, A)=0$ almost everywhere, hence $A \in \operatorname{ker} \omega_{\lambda}$.

The proof is similar for the associated GNS representations. If $A \in \operatorname{ker} \pi_{\omega} \cap \mathfrak{U}_{0}$, then $\left\|\pi_{\omega}(A) f\right\|=0 \quad \forall f \in \mathscr{D} \quad$ implies $\pi_{\omega, \lambda}(A) f(\lambda)=0$ $\forall f(\lambda) \in \mathscr{D}(\lambda)$ such that $f \in \mathscr{D}$, in particular for $f \in \mathscr{D}$. Since we have shown that $\mathscr{D}_{0}(\lambda)$ is a core for every $\pi_{\omega, \lambda}(A)(\S 3.2)$, this implies $\pi_{\omega, \lambda}(A)=0$ hence $A \in \operatorname{ker} \pi_{\omega, \lambda} \cap \mathfrak{A}_{0}$. If now $A \in\left(\mathfrak{A} \backslash \mathfrak{A}_{0}\right) \cap \operatorname{ker} \pi_{\omega}$, there exists a net $\left\{A^{\alpha}\right\}$ in $\mathfrak{U}_{0}$ tending to $A$, and since $\pi_{\omega}$ is a strongly continuous representation, $\left\|\left(\pi_{\omega}\left(A^{\alpha}\right)-\pi_{\omega}(A)\right) f\right\| \rightarrow 0$ for every $f \in \mathscr{D}$. If we restrict ourselves to $f \in \mathscr{D}_{0}$, we may find a sequence $\left\{A^{i}\right\} \subset\left\{A^{\alpha}\right\}$ and a subsequence $\left\{A^{i} k\right.$, independant of $f$. such that $\pi_{\omega}\left(A^{i}\right) f \rightarrow \pi_{\omega}(A) f=0$ and $\pi_{\omega, \lambda}\left(A^{i}\right) f(\lambda) \rightarrow \pi_{\omega, \lambda}(A) f(\lambda)=0$. Since $\mathscr{D}_{0}(\lambda)$ is a core for $\pi_{\omega, \lambda}(A)$, we have that $A \in \operatorname{ker} \pi_{\omega, \lambda}$ for almost every $\lambda \in \Lambda$.

\section{References}

[1] H. J. Borchers, in RCP 25 (Strasbourg) 22 (1975), 26.

[2] J. P. Antoine, W. Karwowski, Publ. RIMS, Kyoto Univ., 21 (1985), 205; Add/Err., ibid 22 (1986).

[3] - in Spontaneous Symmetry Breakdoun and Related Subjects (Proc. Karpacz 1985) pp. 247-267 ; L. Michel, J. Mozrzymas, A. Pekalski (eds)-World Scientific, Singapore 1985.

[4] G. Lassner, Rep. Math. Phys., 3 (1972), 279; Wiss. Z. Karl-Marx universitaet Leipzig, Math.-Naturwiss. R., 24 (1975), 465; and subsequent papers.

[5] A. N. Vasil'ev, Theor. Math. Phys., 2 (1970), 113.

[6] R. T. Powers, Commun. Math. Phys., 21 (1971), 85; Trans. Amer. Math. Soc., 187 (1974), 261. 
[7] R. Ascoli, G. Epifanio, A. Restivo, Commun. Math. Phys., 18 (1970), 291 ; Rivista Mat. Univ. Parma, 3 (1974), 21.

[8] G. G. Emch, H. J. F. Knops, J. Math. Phys., 11 (1970), 3008.

[9] G. Lassner, Physica, A124 (1984), 471.

[10] A. Grossmann, Commun. Math. Phys., 4 (1967), 203.

[11] J. E. Roberts, J. Math. Phys., 7 (1966), 1097; Commun. Math. Phys., 3 (1966), 98.

[12] J. P. Antoine, G. Lassner, preprint in preparation.

[13] See, for instance, J. Dixmier, Les algèbres d'opérateurs dans l'espace hilbertien, GauthierVillars-Paris 1962.

[14] A. E. Nussbaum, Duke Math. J., 31 (1964), 33.

[15] H. J. Borchers, J. Yngvason, Commun. Math. Phys., 42 (1975), 231.

[16] G. C. Hegerfeldt, Commun. Math. Phys., 45 (1975), 133.

[17] F. Debacker-Mathot, Commun. Math. Phys., 71 (1980), 47.

[18] F. Mathot, Pacific J. Math., 90 (1980), 411.

[19] K. Schmuedgen, Rep. Math. Phys., 17 (1980), 359; Acta Math. Acad. Sci. Hung, 35 (1980), 139.

[20] See, for instance, W. Timmermann, preprint E5-85-91 JINR Dubna 1985.

[21] H. Araki, J. P. Jurzak, Publ. RIMS, Kyoto Univ., 18 (1982), 1013.

[22] G. Lassner, Wiss. Z. Karl-Marx-Univ. Leipzig, Math. Naturwzss. R., 30 (1981), 572 ; also in "Algèbres d'opérateurs et leurs applications en physique mathématrque" (Proceedings Marseille 1977), A. Connes, D. Kastler and D. W. Robinson (eds), Editions du CNRS, Paris (1979), 249.

[23] See, for instance, O. Bratelli, D. W. Robinson, Operator algeiras and Quantum Statıstical Me hanics I, Springer, Berlin 1979.

[24] F. Mathot, J. Math. Phys., 26 (1985), 1118.

[25] G. Epifanio, C. Trapani, J. Math. Phys., 25 (1984), 2633.

[26] K. D. Kuersten, Publ. RIMS Kyoto Univ. 22 (1986), 151.

[27] J. P. Antome, F. Mathot, Partial *algebras of closed operators and their commutants, $I$. General siructure, Ann. Inst. H. Poincaré (in press).

[28] S. Gudder, W. Scruggs, Pacific J. Math., 70 (1977), 369.

[29] A. Inoue, Japan J. Math., 9 (1983), 247.

[30] J. P. Antoine, F. Mathot, C. Trapani, Partial *-algebras of closed operators and their commutants, II. Commutants and bicommutants, Ann. Inst. H. Poincaré (inpress). 